\title{
On Refined Neutrosophic Hypervector Spaces
}

\author{
${ }^{1}$ M.A. Ibrahim, ${ }^{2}$ A.A.A. Agboola,${ }^{3}$ B.S. Badmus, ${ }^{4}$ S.A. Akinleye \\ ${ }^{1,2,4}$ Department of Mathematics, Federal University of Agriculture, Abeokuta, Nigeria. \\ ${ }^{3}$ Department of Physics, Federal University of Agriculture, Abeokuta, Nigeria. \\ muritalaibrahim40@gmail.com ${ }^{1}$, agboolaaaa@funaab.edu.ng ${ }^{2}$, badmusbs@yahoo.com ${ }^{3}$, \\ sa_akinleye@yahoo.com ${ }^{4}$
}

\begin{abstract}
This paper presents the refinement of neutrosophic hypervector spaces and studies some of its basic properties. Some basic definitions and important results are presented. The paper also establishes the existence of a good linear transformation between a weak refined neutrosophic hypervector space $V\left(I_{1}, I_{2}\right)$ and a weak neutrosophic hypervector space $V(I)$.

Keywords: Neutrosophy, neutrosophic hypervector space, neutrosophic subhypervector space, refined neutrosophic hypervector space, refined neutrosophic subhypervector space, refined neutrosophic hypervector space homomorphism.
\end{abstract}

\section{Introduction and Preliminaries}

The concept of algebraic hyperstructure was first introduced by Marty [25]. He presented the definition of a hypergroup, studied its properties and applied them to study the groups of rational algebraic functions. Also, Marty used the new approach to solve several problems of the non-commutative algebra. Since then, several researchers have been working on this new field of modern algebra and developed it to a very large extent.

M. Krasner [ $[26]$, introduced the notions of hyperring and hyperfield and used them as technical tools in the study of the approximation of valued fields. There exist several types of hyperrings, some of which are: additive hyperring, multiplicative hyperring and general hyperrings. An important class of additive hyperrings is Krasner hyperrings [232934].

A class of hyperrings $(R,+,$.$) where { }^{\prime \prime}+$ "and "." are hyperoperations was introduced by De Salvo [24]. This class of hyperrings has been further studied by Asokkumar [9], Asokkumar and Velrajan [10|11,28] and Davvaz and Leoreanu-Fotea [23]. Mittas in [27] introduced the theory of canonical hypergroups. J. Mittas was the first who studied them independently from their operations. Some connected hyperstructures with canonical hypergroups were introduced and analyzed by P. Corsini [ ${ }^{21}[22]$, P. Bonansinga [ ${ }^{19}[20]$, and K. Serafimidis in $\left[\begin{array}{ll}32 & 33\end{array}\right]$. Further contributions to the theory of hyperstructures can be found in the books of P. Corsini [21], T. Vougiouklis , P. Corsini and V. Leoreanu [22], and Davvaz and V. Leoreanu [23]. The notion of hypervector spaces was introduced by M. Scafati Tallini. In the definition [31] of hypervector spaces, M. Scafati Tallini considered the field as the usual field. In $\left[{ }^{30}\right]$, Sanjay Roy and T. K. Samanta generalized the notion of hypervector space by considering the hyperfield and considering the multiplication structure of a vector by a scalar as a hyperoperation like M. Scafati Tallini and they both called the hyperstructure a hypervector space. They established basic properties of hypervector space and thereafter the notions of linear combinations, linearly dependence, linearly independence, Hamel basis were introduced and several important properties like deletion theorem, extension theorem were developed.

Neutrosophy is a new branch of philosophy that studies the origin, nature and scope of neutralities, as well as their interactions with different ideational spectra. Neutrosophic set and neutrosophic logic were introduced in 1995 by Smarandache as generalizations of fuzzy logic/set [43] and respectively intuitionistic fuzzy logic/set [13]. In neutrosophic logic, each proposition has a degree of truth $(T)$, a degree of indeterminancy $(I)$ and a degree of falsity $(F)$, where $T, I, F$ are standard or non-standard subsets of $]^{-} 0,1^{+}[$as can be seen in 
[35 36]. A comprehensive review of neutrosophic set, neutrosophic soft set, neutrosophic topological spaces,

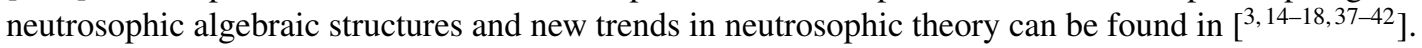

Agboola and Davvaz introduced and studied neutrosophic hypergroups and presented some of their elementary properties in $\left[^{7}\right]$ and in $\left[{ }^{8}\right]$, they studied and presented basic properties of canonical hypergroups and hyperrings in a neutrosophic environment, Quotient neutrosophic canonical hypergroups and neutrosophic hyperrings were also presented. In [5] , Agboola and Akinleye studied neutrosophic hypervector spaces and they presented their basic properties.

In [ [36], Smarandache introduced the concept of refined neutrosophic logic and neutrosophic set which allows for the splitting of the components $\left\langle T, I, F>\right.$ into the form $<T_{1}, T_{2}, \cdots, T_{p} ; I_{1}, I_{2}, \cdot, I_{r}$;

$F_{1}, F_{2}, \cdots, F_{s}>$. This refinement has given rise to the extension of neutrosophic numbers $a+b I$ into refined neutrosophic numbers of the form $\left(a+b_{1} I_{1}+b_{2} I_{2}+\cdots+b_{n} I_{n}\right)$ are real or complex numbers which has led to the introduction of refined neutrosophic set. Refined neutrosophic set has been applied in the development of refined neutrosophic algebraic structures and refined neutrosophic hyperstructures. Agboola in [4] introduced the concept of refined neutrosophic algebraic structures and studied refined neutrosophic groups in particular. Since then, several researchers in this field have studied this concept and a great deal of results have been published. Recently for instance, Adeleke et al published results on refined neutrosophic rings, refined neutrosophic subring in $\left[^{1}\right]$ and in $\left[^{2}\right]$, they presented some results on refined neutrosophic ideals and refined neutrosophic homomorphism. The present paper is devoted to the study of refined neutrosophic hypervector space and presents some elementary properties of this structure.

For the purposes of this paper, it will be assumed that $I$ splits into two indeterminacies $I_{1}$ [contradiction (true $(T)$ and false $(F))$ ] and $I_{2}$ [ignorance (true $(T)$ or false $(F)$ )]. It then follows logically that:

$$
\begin{aligned}
& I_{1} I_{1}=I_{1}^{2}=I_{1}, \\
& I_{2} I_{2}=I_{2}^{2}=I_{2}, \text { and } \\
& I_{1} I_{2}=I_{2} I_{1}=I_{1} .
\end{aligned}
$$

Definition 1.1. Let $(F,+,$.$) be any field. The triple (F(I),+, \cdot)$ is called a neutrosophic field generated by F and I. $(\mathbb{Q}(I),+, \cdot)$ and $(\mathbb{R}(I),+, \cdot)$ are examples of neutrosophic fields.

Definition 1.2. ${ }^{6}$ Let $(V,+,$.$) be any vector space over a field \mathrm{K}$ and let $V(I)=<V \cup I>$ be a neutrosophic set generated by $\mathrm{V}$ and $\mathrm{I}$. The triple $(V(I),+, \cdot)$ is called a weak neutrosophic vector space over a field $K$. If $V(I)$ is a neutrosophic vector space over a neutrosophic field $K(I)$, then $V(I)$ is called a strong neutrosophic vector space. The elements of $V(I)$ are called neutrosophic vectors and the elements of $K(I)$ are called neutrosophic scalars.

If $u=a+b I, v=c+d I \in V(I)$ where $a, b, c$ and $d$ are vectors in $V$ and $\alpha=k+m I \in K(I)$ where $k$ and $m$ are scalars in $K$, then :

$$
u+v=(a+b I)+(c+d I)=(a+c)+(b+d) I,
$$

and

$$
\alpha u=(k+m I) \cdot(a+b I)=k \cdot a+(k \cdot b+m \cdot a+m \cdot b) I .
$$

Definition 1.3. ${ }^{23}$ Let $\mathrm{H}$ be a non-empty set and $\circ: H \times H \longrightarrow P^{*}(H)$ be a hyperoperation. The couple $(H, \circ)$ is called a hypergroupoid. For any two non-empty subsets $\mathrm{A}$ and $\mathrm{B}$ of $\mathrm{H}$ and $x \in H$, we define

$$
A \circ B=\bigcup_{a \in A, b \in B} a \circ b, \quad A \circ x=A \circ\{x\} \quad \text { and } \quad x \circ B=\{x\} \circ B .
$$

Definition 1.4. ${ }^{23}$ A hypergroupoid $(H, \circ)$ is called a semihypergroup if for all $\mathrm{a}, \mathrm{b}, \mathrm{c}$ of $\mathrm{H}$ we have $(a \circ b) \circ c=a \circ(b \circ c)$, which means that

$$
\bigcup_{u \in a \circ b} u \circ c=\bigcup_{v \in b \circ c} a \circ v
$$

A hypergroupoid $(H, \circ)$ is called a quasihypergroup if for all $a \in H$ we have $a \circ H=H \circ a=H$. This condition is also called the reproduction axiom.

Definition 1.5. ${ }^{23}$ A hypergroupoid $(H, \circ)$ which is both a semihypergroup and a quasi- hypergroup is called a hypergroup.

Definition 1.6. ${ }^{23}$ Let $(H, \circ)$ and $\left(H^{\prime}, \circ^{\prime}\right)$ be two hypergroupoids. A map $\phi: H \longrightarrow H^{\prime}$, is called 
1. an inclusion homomorphism if for all $\mathrm{x}$, $\mathrm{y}$ of $\mathrm{H}$, we have $\phi(x \circ y) \subseteq \phi(x) \circ^{\prime} \phi(y)$;

2. a good homomorphism if for all $\mathrm{x}$, $\mathrm{y}$ of $\mathrm{H}$, we have $\phi(x \circ y)=\phi(x) \circ^{\prime} \phi(y)$.

Definition 1.7. ${ }^{23}$ Let $\left(H_{1}, \star_{1}\right)$ and $\left(H_{2}, \star_{2}\right)$ be any two refined hypergroupoids and let $f: H_{1} \longrightarrow H_{2}$ be a map. We say that :

1. $f$ is a homomorphism if for all $x, y$ of $H_{1}$,

$$
f\left(x \star_{1} y\right) \subset f(x) \star_{2} f(y)
$$

2. $f$ is a good homomorphism if for all $x, y$ of $H_{1}$,

$$
f\left(x \star_{1} y\right)=f(x) \star_{2} f(y)
$$

3. $f$ is a strong homomorphism on the left if

$$
f(x) \in f(y) \star_{2} f(z) \Longrightarrow \exists y^{\prime} \in H_{1} \ni f(y)=f\left(y^{\prime}\right) \text { and } x \in y^{\prime} \star_{1} z .
$$

Similarly, we can define a homomorphism, which is strong on the right. If $f$ is strong on the right and on the left we say that $f$ is a strong homomorphism.

Definition 1.8. ${ }^{23}$ Let $\mathrm{H}$ be a non-empty set and let + be a hyperoperation on $\mathrm{H}$. The couple $(H,+)$ is called a canonical hypergroup if the following conditions hold:

1. $x+y=y+x$, for all $x, y \in H$,

2. $x+(y+z)=(x+y)+z$, for all $x, y, z \in H$,

3. there exist a neutral element $0 \in H$ such that $x+0=\{x\}=0+x$, for all $x \in H$,

4. for every $x \in H$, there exist a unique element $-x \in H$ such that $0 \in x+(-x) \cap(-x)+x$,

5. $z \in x+y$ implies $y \in-x+z$ and $x \in z-y$, for all $x, y, z \in H$.

Definition 1.9. ${ }^{23}$ A hyperring is a triple $(R,+, \cdot)$ satisfying the following axioms:

1. $(R,+)$ is a canonical hypergroup.

2. $(R, \cdot)$ is a semihypergroup such that $x \cdot 0=0 \cdot x=0$ for all $x \in R$, that is, 0 is a bilaterally absorbing element.

3. For all $x, y, z \in R$

(a) $x \cdot(y+z)=x \cdot y+x \cdot z$ and

(b) $(x+y) \cdot z=x \cdot z+y \cdot z$.

That is, the hyperoperation $\cdot$ is distributive over the hyperoperation + .

Definition 1.10. ${ }^{5}$ Let $P(V)$ be the power set of a set $V, P^{*}(V)=P(V)-\{\emptyset\}$ and let $K$ be a field. The quadruple $(V,+, \bullet, K)$ is called a hypervector space over a field $K$ if:

1. $(V,+)$ is an abelian group.

2. $\bullet: K \times V \longrightarrow P^{*}(V)$ is a hyperoperation such that for all $k, m \in K$ and $u, v \in V$, the following conditions hold:
(a) $(k+m) \bullet u \subseteq(k \bullet u)+(m \bullet u)$,
(b) $k \bullet(u+v) \subseteq(k \bullet u)+(k \bullet v)$,
(c) $k \bullet(m \bullet u)=(k m) \bullet u$, where $k \bullet(m \bullet u)=\{k \bullet v: v \in m \bullet u\}$,
(d) $(-k) \bullet u=k \bullet(-u)$,
(e) $u \in 1 \bullet u$. 
A hypervector space is said to be strongly left distributive (resp. strongly right distributive) if equality holds in (a) (resp. in (b)). $(V,+, \bullet, K)$ is called a strongly distributive hypervector space if it is both strongly left and strongly right distributive.

Definition 1.11. ${ }^{[12}$ Let $V$ and $W$ be hypervector spaces over $K$. A mapping $T: V \longrightarrow W$ is called

1. weak linear transformation iff

$$
T(x+y)=T(x)+T(y) \text { and } T(a \circ x) \cap a \circ T(x) \neq \emptyset, \forall x, y \in V, a \in K,
$$

2. linear transformation iff

$$
T(x+y)=T(x)+T(y) \text { and } T(a \circ x) \subseteq a \circ T(x), \forall x, y \in V, a \in K,
$$

3. good linear transformation iff

$$
T(x+y)=T(x)+T(y) \text { and } T(a \circ x)=a \circ T(x), \forall y \in V, a \in K .
$$

Definition 1.12. ${ }^{7}$ Let $(H, \star)$ be any hypergroup and let $\langle H \cup I\rangle=\{x=(a, b I): a, b \in H\}$.

The couple $N(H)=(<H \cup I>, \star)$ is called a neutrosophic hypergroup generated by $\mathrm{H}$ and I under the hyperoperation $\star$. The part $a$ is called the non-neutrosophic part of $x$ and the part $b$ is called the neutrosophic part of $x$.

If $x=(a, b I)$ and $y=(c, d I)$ are any two elements of $N(H)$, where $a, b, c, d \in H$, then

$x \star y=(a, b I) \star(c, d I)=\{(u, v I) \mid u \in a \star c, v \in a \star d \cup b \star c \cup b \star d\}=(a \star c,(a \star d \cup b \star c \cup b \star d) I)$. Note that $a \star c \subseteq H$ and $(a \star d \cup b \star c \cup b \star d) \subseteq H$.

Definition 1.13. ${ }^{8}$ A neutrosophic hyperring is a triple $(N(R),+,$.$) satisfying the following axioms :$

1. $(N(R),+)$ is a neutrosophic canonical hypergroup.

2. $(N(R),$.$) is a neutrosophic semihypergroup.$

For all $(a, b I),(c, d I),(e, f I) \in N(R)$,

(a) $(a, b I) \cdot((c, d I)+(e, f I))=(a, b I) \cdot(c, d I)+(a, b I) \cdot(e, f I)$ and

(b) $((c, d I)+(e, f I)) \cdot(a, b I)=(c, d I) \cdot(a, b I)+(e, f I) \cdot(a, b I)$.

Definition 1.14. 6 Let $(V,+, \bullet, K)$ be any strongly distributive hypervector space over a field $K$ and let $V(I)=<V \cup I>=\{u=(a, b I): a, b \in V\}$ be a set generated by $V$ and $I$.

The quadruple $(V(I),+, \bullet, K)$ is called a weak neutrosophic strongly distributive hypervector space over a field $\mathrm{K}$.

For every $u=(a, b I), v=(c, d I) \in V(I)$ and $k \in K$, then

$$
\begin{gathered}
u+v=(a+c,(b+d) I) \in V(I), \\
k \bullet u=\{(x, y I): x \in k \bullet a, y \in k \bullet b\} .
\end{gathered}
$$

If $K$ is a neutrosophic field, that is, $K=K(I)$, then the quadruple $(V(I),+, \bullet, K(I))$ is called a strong neutrosophic strongly distributive hypervector space over a neutrosophic field $K(I)$. For every $u=(a, b I), v=$ $(c, d I) \in V(I)$ and $\alpha=(k, m I) \in K(I)$, we define

$$
\begin{gathered}
u+v=(a+c,(b+d) I) \in V(I), \\
\alpha \bullet u=\{(x, y I): x \in k \bullet a, y \in k \bullet b \cup m \bullet a \cup m \bullet b\} .
\end{gathered}
$$

The zero neutrosophic vector of $V(I),(0,0 I)$, is denoted by $\theta$, the zero element $0 \in K$ is represented by $(0,0 I)$ in $K(I)$ and $1 \in K$ is represented by $(1,0 I)$ in $K(I)$.

Definition 1.15. ${ }^{4}$ If $*: X\left(I_{1}, I_{2}\right) \times X\left(I_{1}, I_{2}\right) \mapsto X\left(I_{1}, I_{2}\right)$ is a binary operation defined on $X\left(I_{1}, I_{2}\right)$, then the couple $\left(X\left(I_{1}, I_{2}\right), *\right)$ is called a refined neutrosophic algebraic structure and it is named according to the laws (axioms) satisfied by $*$. 
Definition 1.16. ${ }^{4}$ Let $\left(X\left(I_{1}, I_{2}\right),+,.\right)$ be any refined neutrosophic algebraic structure where " + " and "." are ordinary addition and multiplication respectively.

For any two elements $\left(a, b I_{1}, c I_{2}\right),\left(d, e I_{1}, f I_{2}\right) \in X\left(I_{1}, I_{2}\right)$, we define

$$
\begin{gathered}
\left(a, b I_{1}, c I_{2}\right)+\left(d, e I_{1}, f I_{2}\right)=\left(a+d,(b+e) I_{1},(c+f) I_{2}\right), \\
\left(a, b I_{1}, c I_{2}\right) \cdot\left(d, e I_{1}, f I_{2}\right)=\left(a d,(a e+b d+b e+b f+c e) I_{1},(a f+c d+c f) I_{2}\right) .
\end{gathered}
$$

Definition 1.17. " If $^{\prime \prime}+{ }^{\prime \prime}$ and ${ }^{\prime \prime} . "$ are ordinary addition and multiplication, $I_{k}$ with $k=1,2$ have the following properties:

1. $I_{k}+I_{k}+\cdots+I_{k}=n I_{k}$.

2. $I_{k}+\left(-I_{k}\right)=0$.

3. $I_{k} \cdot I_{k} \cdots I_{k}=I_{k}^{n}=I_{k}$ for all positive integers $n>1$.

4. $0 \cdot I_{k}=0$.

5. $I_{k}^{-1}$ is undefined and therefore does not exist.

\section{Formulation of Refined Neutrosophic Hypervector Space}

This section shows the formulation of refined neutrosophic hypervector space and present some of its properties.

Definition 2.1. Let $(V,+, \bullet, K)$ be any strongly distributive hypervector space over a field $K$ and let

$$
V\left(I_{1}, I_{2}\right)=<V \cup\left(I_{1}, I_{2}\right)>=\left\{u=\left(a, b I_{1}, c I_{2}\right): a, b, c \in V\right\}
$$

be a set generated by $V, I_{1}$ and $I_{2}$. The quadruple $\left(V\left(I_{1}, I_{2}\right),+, \bullet, K\right)$ is called a weak refined neutrosophic strongly distributive hypervector space over a field $K$.

For every element $u=\left(a, b I_{1}, c I_{2}\right), v=\left(d, e I_{1}, f I_{2}\right) \in V\left(I_{1}, I_{2}\right)$, and $k \in K$ we define

$$
\begin{gathered}
u+v=\left(a+d,(b+e) I_{1},(c+f) I_{2}\right) \in V\left(I_{1}, I_{2}\right), \\
k \bullet u=\left\{\left(x, y I_{1}, z I_{2}\right): x \in k \bullet a, y \in k \bullet b, z \in k \bullet c\right\} .
\end{gathered}
$$

If $K$ is a refined neutrosophic field, that is, $K=K\left(I_{1}, I_{2}\right)$, then the quadruple $\left(V\left(I_{1}, I_{2}\right),+, \bullet, K\left(I_{1}, I_{2}\right)\right)$ is called a strong refined neutrosophic strongly distributive hypervector space over a refined neutrosophic field $K\left(I_{1}, I_{2}\right)$.

For every element $u=\left(a, b I_{1}, c I_{2}\right), v=\left(d, e I_{1}, f I_{2}\right) \in V\left(I_{1}, I_{2}\right)$, and $\alpha=\left(k, m I_{1}, n I_{2}\right) \in K\left(I_{1}, I_{2}\right)$, we define

$$
\begin{gathered}
u+v=\left(a, b I_{1}, c I_{2}\right)+\left(d, e I_{1}, f I_{2}\right)=\left(a+d,(b+e) I_{1},(c+f) I_{2}\right), \\
\alpha \bullet u=\left\{\left(x, y I_{1}, z I_{2}\right):(x \in k \bullet a, y \in k \bullet b \cup m \bullet a \cup m \bullet b \cup m \bullet c \cup n \bullet b, z \in k \bullet c \cup n \bullet a \cup n \bullet c)\right\} .
\end{gathered}
$$

The elements of $V\left(I_{1}, I_{2}\right)$ are called refined neutrosophic vectors and the elements of $K\left(I_{1}, I_{2}\right)$ are called refined neutrosophic scalars. The zero refined neutrosophic vector of $V\left(I_{1}, I_{2}\right),\left(0,0 I_{1}, 0 I_{2}\right)$, is denoted by $\theta$, the zero element $0 \in K$ is represented by $\left(0,0 I_{1}, 0 I_{2}\right)$ in $K\left(I_{1}, I_{2}\right)$ and $1 \in K$ is represented by $\left(1,0 I_{1}, 0 I_{2}\right) \in K\left(I_{1}, I_{2}\right)$.

Example 2.2. 1. Let $\mathrm{r}$ be a fixed positive integer and let

$V=\mathbb{Q}\left(I_{1}, I_{2}\right)(\sqrt{r})=\left\{\left(a, b \sqrt{r} I_{1}, c \sqrt{r} I_{2}\right): a, b, c \in Q, r \in \mathbb{Z}^{+}\right\}$. Then $\mathrm{V}$ is a weak refined neutrosophic strongly distributive hypervector space over $\mathbb{Q}$. If $u=\left(a, b \sqrt{r} I_{1}, c \sqrt{r} I_{2}\right)$ and $v=$ $\left(d, e \sqrt{r} I_{1}, f \sqrt{r} I_{2}\right)$ then $u+v=(a+d),(b+e) \sqrt{r} I_{1},(c+f) \sqrt{r} I_{2}$ is again in $V$.

Also, for $\alpha \in \mathbb{Q}$, then

$$
\alpha \bullet u=\left\{\left(x, y \sqrt{r} I_{1}, z \sqrt{r} I_{2}\right): x \in \alpha \bullet a, y \in \alpha \bullet b, z \in \alpha \bullet c\right\} \in V .
$$


2. Let $V\left(I_{1}, I_{2}\right)=\mathbb{R}\left(I_{1}, I_{2}\right)$ and let $K=\mathbb{R}$. For all $u=\left(a, b I_{1}, c I_{2}\right), v=\left(d, e I_{1}, f I_{2}\right) \in V\left(I_{1}, I_{2}\right)$ and $k \in K$, define:

$$
\begin{gathered}
u+v=\left(a+d,(b+e) I_{1},(c+f) I_{2}\right) \\
k \bullet u=\left\{\left(x, y I_{1}, z I_{2}\right): x \in k \bullet a, y \in k \bullet b, z \in k \bullet c\right\} .
\end{gathered}
$$

Then $\left(V\left(I_{1}, I_{2}\right),+, \bullet, K\right)$ is a weak neutrosophic strongly distributive hypervector space over the field $K$.

Example 2.3. 1. Let $V\left(I_{1}, I_{2}\right)=\mathbb{R}^{3}\left(I_{1}, I_{2}\right)$ and let $K=\mathbb{R}\left(I_{1}, I_{2}\right)$. For all $u=\left(\left(a, b I_{1}, c I_{2}\right),\left(d, e I_{1}, f I_{2}\right),\left(g, h I_{1}, j I_{2}\right)\right), v=\left(\left(a^{\prime}, b^{\prime} I_{1}, c^{\prime} I_{2}\right),\left(d^{\prime}, e^{\prime} I_{1}, f^{\prime} I_{2}\right),\left(g^{\prime}, h^{\prime} I_{1}, j^{\prime} I_{2}\right)\right) \in$ $V\left(I_{1}, I_{2}\right)$ and $\alpha=\left(k, m I_{1}, n I_{2}\right) \in K\left(I_{1}, I_{2}\right)$, define :

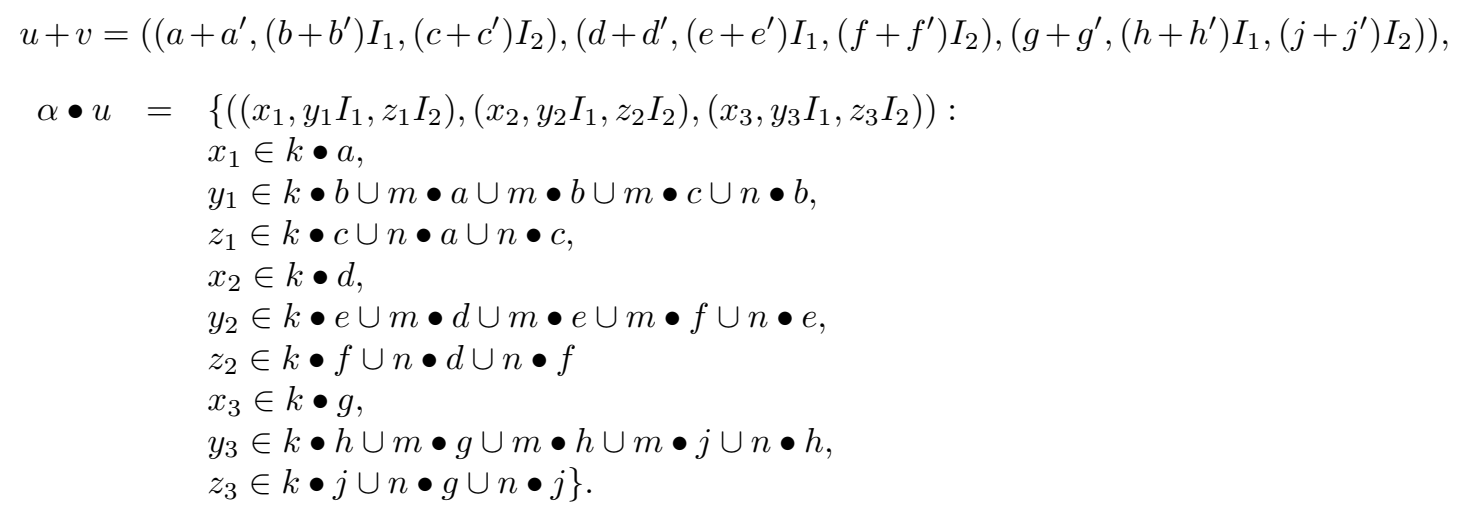

Then $\left(V\left(I_{1}, I_{2}\right),+, \bullet, K\left(I_{1}, I_{2}\right)\right)$ is a strong refined neutrosophic hypervector space over the refined neutrosophic field $K\left(I_{1}, I_{2}\right)$.

2. Let $V\left(I_{1}, I_{2}\right)=\mathbb{R}^{2}\left(I_{1}, I_{2}\right)$ and $K=\mathbb{R}$ define for all $x=(u, v) \in V\left(I_{1}, I_{2}\right)$ with $u=\left(a, b I_{1}, c I_{2}\right)$, $v=\left(d, e I_{1}, f I_{2}\right)$ and $\alpha \in K$

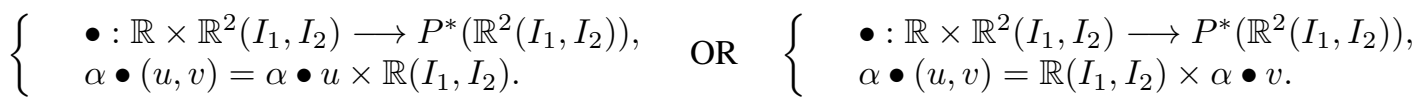

Then $\left(V\left(I_{1}, I_{2}\right),+, \bullet, K\right)$ is a weak refined neutrosophic strongly distributive hypervector space.

From now on, every weak(strong) refined neutrosophic strongly distributive hypervector space will simply be called a weak(strong) refined neutrosophic hypervector space.

Lemma 2.4. Let $V\left(I_{1}, I_{2}\right)$ be a weak refined neutrosophic hypervector space over a field $K$. Then for all $k \in K$ and $u=\left(a, b I_{1}, c I_{2}\right) \in V\left(I_{1}, I_{2}\right)$, we have

1. $k \bullet \theta=\{\theta\}$.

2. $k \bullet u=\{\theta\}$ implies that $k=\theta$ or $u=\theta$.

3. $-u \in(-1) \bullet u$

Proof. 1. $k \bullet \theta=k \bullet(0 \bullet \theta)=(k .0) \bullet \theta=0 \bullet \theta=\theta$

2. Let $k \in K$ and $u \in V$ be such that $k \bullet u=\{\theta\}$.

If $k=0$, then $0 \bullet u=\theta$.

If $k \neq 0$, then $k^{-1} \in K$. Therefore $k \bullet u=\theta \Longrightarrow k^{-1} \bullet(k \bullet u)=k^{-1} \bullet \theta$

$\Longrightarrow\left(k^{-1} \cdot k\right) \bullet u=\theta \Longrightarrow 1_{K} \bullet u=\theta \Longrightarrow u=\theta$.

Proposition 2.5. Every strong refined neutrosophic hypervector space is a weak refined neutrosophic hypervector space.

Proof. Suppose that $V\left(I_{1}, I_{2}\right)$ is a strong refined neutrosophic hypervector space over a refined neutrosophic field $K\left(I_{1}, I_{2}\right)$ say. Since $K \subseteq K\left(I_{1}, I_{2}\right)$ for every field $\mathrm{K}$, then we have that $V\left(I_{1}, I_{2}\right)$ is also a weak refined neutrosophic hypervector space. 
Proposition 2.6. Every weak refined neutrosophic hypervector space is a strongly distributive hypervector space.

Proof. Suppose that $V\left(I_{1}, I_{2}\right)$ is a weak refined neutrosophic hypervector space over a field K. Obviously, $\left(V\left(I_{1}, I_{2}\right),+\right)$ is an abelian group. Let $u=\left(a, b I_{1}, c I_{2}\right), v=\left(d, e I_{1}, f I_{2}\right) \in V\left(I_{1}, I_{2}\right)$ and $k, m \in K$ be arbitrary. Then

$$
\begin{aligned}
k \bullet u+m \bullet u= & \left\{\left(p, q I_{1}, r I_{2}\right): p \in k \bullet a, q \in k \bullet b, r \in k \bullet c\right\}+ \\
& \left\{\left(s, t I_{1}, w I_{2}\right): s \in m \bullet a, t \in m \bullet b, w \in m \bullet c\right\} \\
= & \left\{\left(p+s,(q+t) I_{1},(r+w) I_{2}\right): p+s \in k \bullet a+m \bullet a, q+t \in k \bullet b+m \bullet b,\right. \\
& r+w \in k \bullet c+m \bullet c\} .
\end{aligned}
$$

And,

$(k+m) \bullet u \quad=\left\{\left(x, y I_{1}, z I_{2}\right): x \in(k+m) \bullet a, y \in(k+m) \bullet b, z \in(k+m) \bullet c\right\}$

$=\left\{\left(x, y I_{1}, z I_{2}\right): x \in k \bullet a+m \bullet a, y \in k \bullet b+m \bullet b, z \in k \bullet c+m \bullet c\right\}$

$=k \bullet u+m \bullet u$.

(2) $k \bullet u+k \bullet v=\left\{\left(p, q I_{1}, r I_{2}\right): p \in k \bullet a, q \in k \bullet b, r \in k \bullet c\right\}+$ $\left\{\left(s, t I_{1}, w I_{2}\right): s \in k \bullet d, t \in k \bullet e, w \in k \bullet f\right\}$

$=\left\{\left(p+s,(q+t) I_{1},(r+w) I_{2}\right): p+s \in k \bullet a+k \bullet d, q+t \in k \bullet b+k \bullet e\right.$, $r+w \in k \bullet c+k \bullet f\}$.

And,

$k \bullet(u+v)$

$$
\begin{aligned}
& =k \bullet\left(a+d,(b+e) I_{1},(c+f) I_{2}\right) \\
& =\left\{\left(x, y I_{1}, z I_{2}\right): x \in k \bullet(a+d), y \in k \bullet(b+e), z \in k \bullet(c+f)\right\} \\
& =\left\{\left(x, y I_{1}, z I_{2}\right): x \in k \bullet a+k \bullet d, y \in k \bullet b+k \bullet e, z \in k \bullet c+k \bullet f\right\} \\
& =k \bullet u+k \bullet v .
\end{aligned}
$$

(3) $k \bullet(m \bullet u)=k \bullet\left\{\left(x, y I_{1}, z I_{2}\right): x \in m \bullet a, y \in m \bullet b, z \in m \bullet c\right\}$

$=\left\{\left(p, q I_{1}, r I_{2}\right): p \in k \bullet x, q \in k \bullet y, r \in k \bullet z\right\}$

$=\left\{\left(p, q I_{1}, r I_{2}\right): p \in k \bullet(m \bullet a), q \in k \bullet(m \bullet b), r \in k \bullet(m \bullet c)\right\}$

$=\left\{\left(p, q I_{1}, r I_{2}\right): p \in(k m) \bullet a, q \in(k m) \bullet b, r \in(k m) \bullet c\right\}$$$
=(k m) \bullet\left(a, b I_{1}, c I_{2}\right)
$$$$
=(k m) \bullet u \text {. }
$$

(4) $(-k) \bullet u=\left\{\left(x, y I_{1}, z I_{2}\right): x \in(-k) \bullet a, y \in(-k) \bullet b, z \in(-k) \bullet c\right\}$

$=\left\{\left(x, y I_{1}, c I_{2}\right): x \in k \bullet(-a), y \in k \bullet(-b), z \in k \bullet(-c)\right\}$

$=k \bullet\left(-a,-b I_{1},-c I_{2}\right)$

$=k \bullet(-u)$.

(5) $1 \bullet u=\left\{\left(x, y I_{1}, z I_{2}\right): x \in 1 \bullet a, y \in 1 \bullet b, z \in 1 \bullet c\right\}$

$=\left\{\left(x, y I_{1}, z I_{2}\right): x \in\{a\}, y \in\{b\}, z \in\{c\}\right\}$

$=\left\{\left(a, b I_{1}, c I_{2}\right)\right\}$.

$\Longrightarrow u \in 1 \bullet u$.

Accordingly, $V\left(I_{1}, I_{2}\right)$ is a strongly distributive hypervector space.

Corollary 2.7. Every weak refined neutrosophic hypervector space which is strongly right distributive is strongly left distributive.

Proof. The proof follows from the proof of Proposition 2.6.

Proposition 2.8. Let $\left(V_{1}\left(I_{1}, I_{2}\right),+_{1}, \bullet_{1}, K\left(I_{1}, I_{2}\right)\right)$ and $\left(V_{2}\left(I_{1}, I_{2}\right),+_{2}, \bullet_{2}, K\left(I_{1}, I_{2}\right)\right)$ be two strong refined neutrosophic hypervector spaces over a refined neutrosophic field $K\left(I_{1}, I_{2}\right)$. Let

$V_{1}\left(I_{1}, I_{2}\right) \times V_{2}\left(I_{1}, I_{2}\right)=$

$\left\{\left(\left(a_{1}, b_{1} I_{1}, c_{1} I_{2}\right),\left(a_{2}, b_{2} I_{1}, c_{2} I_{2}\right)\right):\left(a_{1}, b_{1} I_{1}, c_{1} I_{2}\right) \in V_{1}\left(I_{1}, I_{2}\right),\left(a_{2}, b_{2} I_{1}, c_{2} I_{2}\right) \in V_{2}\left(I_{1}, I_{2}\right)\right\}$ and for all $u=\left(\left(a_{1}, b_{1} I_{1}, c I_{2}\right),\left(a_{2}, b_{2} I_{1}, c_{2} I_{2}\right)\right), v=\left(\left(a_{1}^{\prime}, b_{1}^{\prime} I_{1}, c_{1}^{\prime} I_{2}\right),\left(a_{2}^{\prime}, b_{2}^{\prime} I_{2}, c_{2}^{\prime} I_{2}\right)\right) \in V_{1}\left(I_{1}, I_{2}\right) \times V_{2}\left(I_{1}, I_{2}\right)$ and

$\alpha=\left(k, m I_{1}, n I_{2}\right) \in K\left(I_{1}, I_{2}\right)$, define:

$u+v=\left(\left(\left(a_{1}+a_{1}^{\prime}\right),\left(b_{1}+b_{1}^{\prime}\right) I_{1},\left(c_{1}+c_{1}^{\prime}\right) I_{2}\right),\left(\left(a_{2}+a_{2}^{\prime}\right),\left(b_{2}+b_{2}^{\prime}\right) I_{1},\left(c_{2}+c_{2}^{\prime}\right) I_{2}\right)\right)$,

$\alpha \bullet u=\left\{\left(\left(x, y I_{1}, z I_{2}\right),\left(p, q I_{1}, r I_{2}\right)\right)\right\}$.

$x \in k \bullet a_{1}$,

$y \in k \bullet b_{1} \cup m \bullet a_{1} \cup m \bullet b_{1} \cup m \bullet c_{1} \cup n \bullet b_{1}$,

$z \in k \bullet c_{1} \cup n \bullet a_{1} \cup n \bullet c_{1}$, 
$p \in k \bullet a_{2}$,

$q \in k \bullet b_{2} \cup m \bullet a_{2} \cup m \bullet b_{2} \cup m \bullet c_{2} \cup n \bullet b_{2}$,

$z \in k \bullet c_{2} \cup n \bullet a_{2} \cup n \bullet c_{2}$.

Then $\left(V_{1}\left(I_{1}, I_{2}\right) \times V_{2}\left(I_{1}, I_{2}\right),+, \bullet, K\left(I_{1}, I_{2}\right)\right)$ is a strong neutrosophic hypervector space.

Proof. Suppose that $V_{1}\left(I_{1}, I_{2}\right)$ and $V_{2}\left(I_{1}, I_{2}\right)$ are strong refined neutrosophic hypervector spaces over a refined neutrosophic field $K\left(I_{1}, I_{2}\right)$.

Let $u=\left(\left(a_{1}, b_{1} I_{1}, c_{1} I_{2}\right),\left(a_{2}, b_{2} I_{1}, c_{2} I_{2}\right)\right), v=\left(\left(a_{1}^{\prime}, b_{1}^{\prime} I_{1}, c_{1}^{\prime} I_{2}\right),\left(a_{2}^{\prime}, b_{2}^{\prime} I_{2}, c_{2}^{\prime} I_{2}\right)\right) \in V_{1}\left(I_{1}, I_{2}\right) \times V_{2}\left(I_{1}, I_{2}\right)$ and $\alpha=\left(k, m I_{1}, n I_{2}\right), \beta=\left(k^{\prime}, m^{\prime} I_{1}, n^{\prime} I_{2}\right) \in K\left(I_{1}, I_{2}\right)$ be arbitrary.

1. We can easily show that $\left(V_{1}\left(I_{1}, I_{2}\right) \times V_{2}\left(I_{1}, I_{2}\right),+\right)$ is an abelian group.

2. Now we want to show that $(\alpha+\beta) \bullet u \subseteq \alpha \bullet u+\beta \bullet u$.

Consider

$(\alpha+\beta) \bullet u=\left(k+k^{\prime},\left(m+m^{\prime}\right) I_{1},\left(n+n^{\prime}\right) I_{2}\right) \bullet\left(\left(a_{1}, b_{1} I_{1}, c_{1} I_{2}\right),\left(a_{2}, b_{2} I_{1}, c_{2} I_{2}\right)\right)$

$\subseteq\left\{\left(\left(x, y I_{1}, z I_{2}\right),\left(p, q I_{1}, r I_{2}\right)\right): x \in\left(k+k^{\prime}\right) \bullet a_{1}, y \in\left(k+k^{\prime}\right) \bullet b_{1} \cup\left(m+m^{\prime}\right) \bullet a_{1} \cup\left(m+m^{\prime}\right) \bullet\right.$

$b_{1} \cup\left(m+m^{\prime}\right) \bullet c_{1} \cup\left(n+n^{\prime}\right) \bullet b_{1}, z \in\left(k+k^{\prime}\right) \bullet c_{1} \cup\left(n+n^{\prime}\right) \bullet a_{1} \cup\left(n+n^{\prime}\right) \bullet c_{1}$,

$p \in\left(k+k^{\prime}\right) \bullet a_{2}, q \in\left(k+k^{\prime}\right) \bullet b_{2} \cup\left(m+m^{\prime}\right) \bullet a_{2} \cup\left(m+m^{\prime}\right) \bullet b_{2} \cup\left(m+m^{\prime}\right) \bullet c_{2} \cup\left(n+n^{\prime}\right) \bullet b_{2}, z \in$ $\left.\left(k+k^{\prime}\right) \bullet c_{2} \cup\left(n+n^{\prime}\right) \bullet a_{2} \cup\left(n+n^{\prime}\right) \bullet c_{2}\right\}$

$=\left\{\left(\left(x, y I_{1}, z I_{2}\right),\left(p, q I_{1}, r I_{2}\right)\right): x \in k \bullet a_{1}+k^{\prime} \bullet a_{1}, y \in k \bullet b_{1}+k^{\prime} \bullet b_{1} \cup m \bullet a_{1}+m^{\prime} \bullet a_{1} \cup m \bullet b_{1}+\right.$ $m^{\prime} \bullet b_{1} \cup m \bullet c_{1}+m^{\prime} \bullet c_{1} \cup n \bullet b_{1}+n^{\prime} \bullet b_{1}, z \in k \bullet c_{1}+k^{\prime} \bullet c_{1} \cup n \bullet a_{1}+n^{\prime} \bullet a_{1} \cup n \bullet c_{1}+n^{\prime} \bullet c_{1}$, $p \in k \bullet a_{2}+k^{\prime} \bullet a_{2}, q \in k \bullet b_{2}+k^{\prime} \bullet b_{2} \cup m \bullet a_{2}+m^{\prime} \bullet a_{2} \cup m \bullet b_{2}+m^{\prime} \bullet b_{2} \cup m \bullet c_{2}+m^{\prime} \bullet c_{2} \cup n \bullet b_{2}+n^{\prime} \bullet b_{2}, z \in$ $\left.k \bullet c_{2}+k^{\prime} \bullet c_{2} \cup n \bullet a_{2}+n^{\prime} \bullet a_{2} \cup n \bullet c_{2}+n^{\prime} \bullet c_{2}\right\}$.

Now if we take $x=s_{1}+s_{1}^{\prime}, y=t_{1}+t_{1}^{\prime}, z=w_{1}+w_{1}^{\prime}, p=s_{2}+s_{2}^{\prime}, q=t_{2}+t_{2}^{\prime}$ and $r=w_{1}+w_{2}^{\prime}$ then we have

$\left\{\left(\left(s_{1}+s_{1}^{\prime},\left(t_{1}+t_{1}^{\prime}\right) I_{1},\left(w_{1}+w_{1}^{\prime}\right) I_{2}\right),\left(s_{2}+s_{2}^{\prime},\left(t_{2}+t_{2}^{\prime}\right) I_{1},\left(w_{1}+w_{1}^{\prime}\right) I_{2}\right)\right): s_{1}+s_{1}^{\prime} \in k \bullet a_{1}+k^{\prime} \bullet a_{1}, t_{1}+\right.$ $t_{1}^{\prime} \in k \bullet b_{1}+k^{\prime} \bullet b_{1} \cup m \bullet a_{1}+m^{\prime} \bullet a_{1} \cup m \bullet b_{1}+m^{\prime} \bullet b_{1} \cup m \bullet c_{1}+m^{\prime} \bullet c_{1} \cup n \bullet b_{1}+n^{\prime} \bullet b_{1}, w_{1}+w_{1}^{\prime} \in$ $k \bullet c_{1}+k^{\prime} \bullet c_{1} \cup n \bullet a_{1}+n^{\prime} \bullet a_{1} \cup n \bullet c_{1}+n^{\prime} \bullet c_{1}$,

$s_{2}+s_{2}^{\prime} \in k \bullet a_{2}+k^{\prime} \bullet a_{2}, t_{2}+t_{2}^{\prime} \in k \bullet b_{2}+k^{\prime} \bullet b_{2} \cup m \bullet a_{2}+m^{\prime} \bullet a_{2} \cup m \bullet b_{2}+m^{\prime} \bullet b_{2} \cup m \bullet c_{2}+m^{\prime} \bullet c_{2} \cup n \bullet b_{2}+$ $\left.n^{\prime} \bullet b_{2}, w_{2}+w_{2}^{\prime} \in k \bullet c_{2}+k^{\prime} \bullet c_{2} \cup n \bullet a_{2}+n^{\prime} \bullet a_{2} \cup n \bullet c_{2}+n^{\prime} \bullet c_{2}\right\}=\left\{\left(\left(s_{1}, t_{1} I_{1}, w_{1} I_{2}\right),\left(s_{2}, t_{2} I_{1}, w_{2} I_{2}\right)\right):\right.$ $s_{1} \in k \bullet a_{1}, t_{1} \in k \bullet b_{1} \cup m \bullet a_{1} \cup m \bullet b_{1} \cup m \bullet c_{1} \cup n \bullet b_{1}, w_{1} \in k \bullet c_{1} \cup n \bullet a_{1} \cup n \bullet c_{1}, \quad s_{2} \in k \bullet a_{2}, t_{2} \in$ $\left.k \bullet b_{2} \cup m \bullet a_{2} \cup m \bullet b_{2} \cup m \bullet c_{2} \cup n \bullet b_{2}, w_{2} \in k \bullet c_{2} \cup n \bullet a_{2} \cup n \bullet c_{2}\right\}+\left\{\left(\left(s_{1}^{\prime}, t_{1}^{\prime} I_{1}, w_{1}^{\prime} I_{2}\right),\left(s_{2}^{\prime}, t_{2}^{\prime} I_{1}, w_{2}^{\prime} I_{2}\right)\right):\right.$ $s_{1}^{\prime} \in k^{\prime} \bullet a_{1}, t_{1}^{\prime} \in k^{\prime} \bullet b_{1} \cup m^{\prime} \bullet a_{1} \cup m^{\prime} \bullet b_{1} \cup m^{\prime} \bullet c_{1} \cup n^{\prime} \bullet b_{1}, w_{1}^{\prime} \in k^{\prime} \bullet c_{1} \cup n^{\prime} \bullet a_{1} \cup n^{\prime} \bullet c_{1}$, $\left.s_{2}^{\prime} \in k^{\prime} \bullet a_{2}, t_{2}^{\prime} \in k^{\prime} \bullet b_{2} \cup m^{\prime} \bullet a_{2} \cup m^{\prime} \bullet b_{2} \cup m^{\prime} \bullet c_{2} \cup n^{\prime} \bullet b_{2}, w_{2}^{\prime} \in k^{\prime} \bullet c_{2} \cup n^{\prime} \bullet a_{2} \cup n^{\prime} \bullet c_{2}\right\}$ $\subseteq \alpha \bullet u+\beta \bullet u$.

Then $(\alpha+\beta) \bullet u \subseteq \alpha \bullet u+\beta \bullet u$.

3. Now we want to show that $\alpha \bullet(u+v) \subseteq \alpha \bullet u+\alpha \bullet v$ $\alpha \bullet(u+v)=\left(k, m I_{1}, n I_{2}\right) \bullet\left(\left(a_{1}+a_{1}^{\prime},\left(b_{1}+b_{1}^{\prime}\right) I_{1},\left(c_{1}+c_{1}^{\prime}\right) I_{2}\right),\left(a_{2}+a_{2}^{\prime},\left(b_{2}+b_{2}^{\prime}\right) I_{1},\left(c_{2}+c_{2}^{\prime}\right) I_{2}\right)\right)$ $\subseteq\left\{\left(x, y I_{1}, z I_{2}\right),\left(p, q I_{1}, r I_{2}\right): x \in k \bullet\left(a_{1}+a_{1}^{\prime}\right), y \in k \bullet\left(b_{1}+b_{1}^{\prime}\right) \cup m \bullet\left(a_{1}+a_{1}^{\prime}\right) \cup m \bullet\left(b_{1}+b_{1}^{\prime}\right) \cup\right.$ $m \bullet\left(c_{1}+c_{1}^{\prime}\right) \cup n \bullet\left(b_{1}+b_{1}^{\prime}\right), z \in k \bullet\left(c_{1}+c_{1}^{\prime}\right) \cup n \bullet\left(a_{1}+a_{1}^{\prime}\right) \cup n \bullet\left(c_{1}+c_{1}^{\prime}\right)$,

$p \in k \bullet\left(a_{2}+a_{2}^{\prime}\right), q \in k \bullet\left(b_{2}+b_{2}^{\prime}\right) \cup m \bullet\left(a_{2}+a_{2}^{\prime}\right) \cup m \bullet\left(b_{2}+b_{2}^{\prime}\right) \cup m \bullet\left(c_{2}+c_{2}^{\prime}\right) \cup n \bullet\left(b_{2}+b_{2}^{\prime}\right)$, $\left.r \in k \bullet\left(c_{2}+c_{2}^{\prime}\right) \cup n \bullet\left(a_{2}+a_{2}^{\prime}\right) \cup n \bullet\left(c_{2}+c_{2}^{\prime}\right)\right\}$

$=\left\{\left(x, y I_{1}, z I_{2}\right),\left(p, q I_{1}, r I_{2}\right): x \in k \bullet a_{1}+k \bullet a_{1}^{\prime}, y \in k \bullet b_{1}+k \bullet b_{1}^{\prime} \cup m \bullet a_{1}+m \bullet a_{1}^{\prime} \cup\right.$ $m \bullet b_{1}+m \bullet b_{1}^{\prime} \cup m \bullet c_{1}+m \bullet c_{1}^{\prime} \cup n \bullet b_{1}+n \bullet b_{1}^{\prime}, z \in k \bullet c_{1}+k \bullet c_{1}^{\prime} \cup n \bullet a_{1}+n \bullet a_{1}^{\prime} \cup n \bullet c_{1}+n \bullet c_{1}^{\prime}$, $p \in k \bullet a_{2}+k \bullet a_{2}^{\prime}, q \in k \bullet b_{2}+k \bullet b_{2}^{\prime} \cup m \bullet a_{2}+m \bullet a_{2}^{\prime} \cup m \bullet b_{2}+m \bullet b_{2}^{\prime} \cup m \bullet c_{2}+m \bullet c_{2}^{\prime} \cup n \bullet b_{2}+n \bullet b_{2}^{\prime}, r \in$ $\left.k \bullet c_{2}+k \bullet c_{2}^{\prime} \cup n \bullet a_{2}+n \bullet a_{2}^{\prime} \cup n \bullet c_{2}+n \bullet c_{2}^{\prime}\right\}$.

If we take $x=s_{1}+s_{1}^{\prime}, y=t_{1}+t_{1}^{\prime}, z=w_{1}+w_{1}^{\prime}, p=s_{2}+s_{2}^{\prime}, q=t_{2}+t_{2}^{\prime}$ and $r=w_{1}+w_{2}^{\prime}$ then we have

$\left\{\left(s_{1}+s_{1}^{\prime},\left(t_{1}+t_{1}^{\prime}\right) I_{1},\left(w_{1}+w_{1}^{\prime}\right) I_{2}\right),\left(s_{2}+s_{2}^{\prime},\left(t_{2}+t_{2}^{\prime}\right) I_{1},\left(w_{2}+w_{2}^{\prime}\right) I_{2}\right): s_{1}+s_{1}^{\prime} \in k \bullet a_{1}+k \bullet a_{1}^{\prime}, t_{1}+t_{1}^{\prime} \in\right.$ $k \bullet b_{1}+k \bullet b_{1}^{\prime} \cup m \bullet a_{1}+m \bullet a_{1}^{\prime} \cup m \bullet b_{1}+m \bullet b_{1}^{\prime} \cup m \bullet c_{1}+m \bullet c_{1}^{\prime} \cup n \bullet b_{1}+n \bullet b_{1}^{\prime}, w_{1}+w_{1}^{\prime} \in$ $k \bullet c_{1}+k \bullet c_{1}^{\prime} \cup n \bullet a_{1}+n \bullet a_{1}^{\prime} \cup n \bullet c_{1}+n \bullet c_{1}^{\prime}, s_{2}+s_{2}^{\prime} \in k \bullet a_{2}+k \bullet a_{2}^{\prime}, t_{2}+t_{2}^{\prime} \in k \bullet b_{2}+k \bullet b_{2}^{\prime} \cup m \bullet a_{2}+m \bullet$ $\left.a_{2}^{\prime} \cup m \bullet b_{2}+m \bullet b_{2}^{\prime} \cup m \bullet c_{2}+m \bullet c_{2}^{\prime} \cup n \bullet b_{2}+n \bullet b_{2}^{\prime}, w_{2}+w_{2}^{\prime} \in k \bullet c_{2}+k \bullet c_{2}^{\prime} \cup n \bullet a_{2}+n \bullet a_{2}^{\prime} \cup n \bullet c_{2}+n \bullet c_{2}^{\prime}\right\}$ $=\left\{\left(s_{1}, t_{1} I_{1}, w_{1} I_{2}\right),\left(s_{2}, t_{2} I_{1}, w_{2} I_{2}\right): s_{1} \in k \bullet a_{1}, t_{1} \in k \bullet b_{1} \cup m \bullet a_{1} \cup m \bullet b_{1} \cup m \bullet c_{1} \cup n \bullet b_{1}, w_{1} \in\right.$ $k \bullet c_{1} \cup n \bullet a_{1} \cup n \bullet c_{1}, s_{2} \in k \bullet a_{2}, t_{2} \in k \bullet b_{2} \cup m \bullet a_{2} \cup m \bullet b_{2} \cup m \bullet c_{2} \cup n \bullet b_{2}, w_{2} \in k \bullet c_{2} \cup n \bullet a_{2} \cup n \bullet$ $\left.c_{2}\right\}+\left\{\left(s_{1}^{\prime}, t_{1}^{\prime} I_{1}, w_{1}^{\prime} I_{2}\right),\left(s_{2}^{\prime}, t_{2}^{\prime} I_{1}, w_{2}^{\prime} I_{2}\right): s_{1}^{\prime} \in k \bullet a_{1}^{\prime}, t_{1}^{\prime} \in k \bullet b_{1}^{\prime} \cup m \bullet a_{1}^{\prime} \cup m \bullet b_{1}^{\prime} \cup m \bullet c_{1}^{\prime} \cup n \bullet b_{1}^{\prime}, w_{2}^{\prime} \in\right.$ $\left.k \bullet c_{1}^{\prime} \cup n \bullet a_{1}^{\prime} \cup n \bullet c_{1}^{\prime}, s_{2}^{\prime} \in k \bullet a_{2}^{\prime}, t_{2}^{\prime} \in k \bullet b_{2}^{\prime} \cup m \bullet a_{2}^{\prime} \cup m \bullet b_{2}^{\prime} \cup m \bullet c_{2}^{\prime} \cup n \bullet b_{2}^{\prime}, w_{2}^{\prime} \in k \bullet c_{2}^{\prime} \cup n \bullet a_{2}^{\prime} \cup n \bullet c_{2}^{\prime}\right\} \subseteq$ $\alpha \bullet u+\alpha \bullet v$.

Then we have that $\alpha \bullet(u+v) \subseteq \alpha \bullet u+\alpha \bullet v$. 
4. $\alpha \bullet(\beta \bullet u)=\alpha \bullet\left\{\left(\left(x, y I_{1}, z I_{2}\right),\left(p, q I_{1}, r I_{2}\right)\right): x \in k^{\prime} \bullet a_{1}, y \in k^{\prime} \bullet b_{1} \cup m^{\prime} \bullet a_{1} \cup m^{\prime} \bullet b_{1} \cup m^{\prime} \bullet c_{1} \cup\right.$ $n^{\prime} \bullet b_{1}, z \in k^{\prime} \bullet c_{1} \cup n^{\prime} \bullet a_{1} \cup n^{\prime} \bullet c_{1}, p \in k^{\prime} \bullet a_{2}, q \in k^{\prime} \bullet b_{2} \cup m^{\prime} \bullet a_{2} \cup m^{\prime} \bullet b_{2} \cup m^{\prime} \bullet c_{2} \cup n^{\prime} \bullet b_{2}, r \in$ $\left.k^{\prime} \bullet c_{2} \cup n^{\prime} \bullet a_{2} \cup n^{\prime} \bullet c_{2}\right\}$

$=\left\{\left(\left(x^{\prime}, y^{\prime} I_{1}, z^{\prime} I_{2}\right),\left(p^{\prime}, q^{\prime} I_{1}, r^{\prime} I_{2}\right)\right): x^{\prime} \in k \bullet x, y^{\prime} \in k \bullet y \cup m \bullet x \cup m \bullet y \cup m \bullet z \cup n \bullet y\right.$,

$z^{\prime} \in k \bullet z \cup n \bullet x \cup n \bullet z, \quad p^{\prime} \in k \bullet p, q^{\prime} \in k \bullet q \cup m \bullet p \cup m \bullet q \cup m \bullet r \cup n \bullet q$,

$\left.z^{\prime} \in k \bullet r \cup n \bullet p \cup n \bullet r\right\}$

$=\left\{\left(\left(x^{\prime}, y^{\prime} I_{1}, z^{\prime} I_{2}\right),\left(p^{\prime}, q^{\prime} I_{1}, r^{\prime} I_{2}\right)\right): x^{\prime} \in k \bullet\left(k^{\prime} \bullet a_{1}\right), y^{\prime} \in k \bullet\left(k^{\prime} \bullet b_{1} \cup m^{\prime} \bullet a_{1} \cup m^{\prime} \bullet b_{1} \cup m^{\prime} \bullet\right.\right.$ $\left.c_{1} \cup n^{\prime} \bullet b_{1}\right) \cup m \bullet\left(k^{\prime} \bullet a_{1}\right) \cup m \bullet\left(k^{\prime} \bullet b_{1} \cup m^{\prime} \bullet a_{1} \cup m^{\prime} \bullet b_{1} \cup m^{\prime} \bullet c_{1} \cup n^{\prime} \bullet b_{1}\right) \cup m \bullet\left(k^{\prime} \bullet c_{1} \cup n^{\prime} \bullet\right.$ $\left.a_{1} \cup n^{\prime} \bullet c_{1}\right) \cup n \bullet\left(k^{\prime} \bullet b_{1} \cup m^{\prime} \bullet a_{1} \cup m^{\prime} \bullet b_{1} \cup m^{\prime} \bullet c_{1} \cup n^{\prime} \bullet b_{1}\right)$,

$z^{\prime} \in k \bullet\left(k^{\prime} \bullet c_{1} \cup n^{\prime} \bullet a_{1} \cup n^{\prime} \bullet c_{1}\right) \cup n \bullet\left(k^{\prime} \bullet a_{1}\right) \cup n \bullet\left(k^{\prime} \bullet c_{1} \cup n^{\prime} \bullet a_{1} \cup n^{\prime} \bullet c_{1}\right), \quad p^{\prime} \in k \bullet\left(k^{\prime} \bullet a_{2}\right), q^{\prime} \in$ $k \bullet\left(k^{\prime} \bullet b_{2} \cup m^{\prime} \bullet a_{2} \cup m^{\prime} \bullet b_{2} \cup m^{\prime} \bullet c_{2} \cup n^{\prime} \bullet b_{2}\right) \cup m \bullet\left(k^{\prime} \bullet a_{2}\right) \cup m \bullet\left(k^{\prime} \bullet b_{2} \cup m^{\prime} \bullet a_{2} \cup m^{\prime} \bullet b_{2} \cup\right.$ $\left.m^{\prime} \bullet c_{2} \cup n^{\prime} \bullet b_{2}\right) \cup m \bullet\left(k^{\prime} \bullet c_{2} \cup n^{\prime} \bullet a_{2} \cup n^{\prime} \bullet c_{2}\right) \cup n \bullet\left(k^{\prime} \bullet b_{2} \cup m^{\prime} \bullet a_{2} \cup m^{\prime} \bullet b_{2} \cup m^{\prime} \bullet c_{2} \cup n^{\prime} \bullet b_{2}\right)$, $\left.z^{\prime} \in k \bullet\left(k^{\prime} \bullet c_{2} \cup n^{\prime} \bullet a_{2} \cup n^{\prime} \bullet c_{2}\right) \cup n \bullet\left(k^{\prime} \bullet a_{2}\right) \cup n \bullet\left(k^{\prime} \bullet c_{2} \cup n^{\prime} \bullet a_{2} \cup n^{\prime} \bullet c_{2}\right)\right\}$

$=\left\{\left(\left(x^{\prime}, y^{\prime} I_{1}, z^{\prime} I_{2}\right),\left(p^{\prime}, q^{\prime} I_{1}, r^{\prime} I_{2}\right)\right): x^{\prime} \in\left(k k^{\prime}\right) \bullet a_{1}, y^{\prime} \in\left(k k^{\prime}\right) \bullet b_{1} \cup\left(k m^{\prime}\right) \bullet a_{1} \cup\left(k m^{\prime}\right) \bullet b_{1} \cup\left(k m^{\prime}\right) \bullet\right.$ $c_{1} \cup\left(k n^{\prime}\right) \bullet b_{1} \cup\left(m k^{\prime}\right) \bullet a_{1} \cup\left(m k^{\prime}\right) \bullet b_{1} \cup\left(m m^{\prime}\right) \bullet a_{1} \cup\left(m m^{\prime}\right) \bullet b_{1} \cup\left(m m^{\prime}\right) \bullet c_{1} \cup\left(m n^{\prime}\right) \bullet b_{1} \cup\left(m k^{\prime}\right) \bullet$ $c_{1} \cup\left(m n^{\prime}\right) \bullet a_{1} \cup\left(m n^{\prime}\right) \bullet c_{1} \cup\left(n k^{\prime}\right) \bullet b_{1} \cup\left(n m^{\prime}\right) \bullet a_{1} \cup\left(n m^{\prime}\right) \bullet b_{1} \cup\left(n m^{\prime}\right) \bullet c_{1} \cup\left(n n^{\prime}\right) \bullet b_{1}, z^{\prime} \in$ $\left(k k^{\prime}\right) \bullet c_{1} \cup\left(k n^{\prime}\right) \bullet a_{1} \cup\left(k n^{\prime}\right) \bullet c_{1} \cup\left(n k^{\prime}\right) \bullet a_{1} \cup\left(n k^{\prime}\right) \bullet c_{1} \cup\left(n n^{\prime}\right) \bullet a_{1} \cup\left(n n^{\prime}\right) \bullet c_{1}, \quad p^{\prime} \in\left(k k^{\prime}\right) \bullet a_{2}, q^{\prime} \in$ $\left(k k^{\prime}\right) \bullet b_{2} \cup\left(k m^{\prime}\right) \bullet a_{2} \cup\left(k m^{\prime}\right) \bullet b_{2} \cup\left(k m^{\prime}\right) \bullet c_{2} \cup\left(k n^{\prime}\right) \bullet b_{2} \cup\left(m k^{\prime}\right) \bullet a_{2} \cup\left(m k^{\prime}\right) \bullet b_{2} \cup\left(m m^{\prime}\right) \bullet a_{2} \cup$ $\left(m m^{\prime}\right) \bullet b_{2} \cup\left(m m^{\prime}\right) \bullet c_{2} \cup\left(m n^{\prime}\right) \bullet b_{2} \cup\left(m k^{\prime}\right) \bullet c_{2} \cup\left(m n^{\prime}\right) \bullet a_{2} \cup\left(m n^{\prime}\right) \bullet c_{2} \cup\left(n k^{\prime}\right) \bullet b_{2} \cup\left(n m^{\prime}\right) \bullet$ $a_{2} \cup\left(n m^{\prime}\right) \bullet b_{2} \cup\left(n m^{\prime}\right) \bullet c_{2} \cup\left(n n^{\prime}\right) \bullet b_{2}$,

$\left.z^{\prime} \in\left(k k^{\prime}\right) \bullet c_{2} \cup\left(k n^{\prime}\right) \bullet a_{2} \cup\left(k n^{\prime}\right) \bullet c_{2} \cup\left(n k^{\prime}\right) \bullet a_{2} \cup\left(n k^{\prime}\right) \bullet c_{2} \cup\left(n n^{\prime}\right) \bullet a_{2} \cup\left(n n^{\prime}\right) \bullet c_{2}\right\}$

$=\left(\left(k, m I_{1}, n I_{2}\right)\left(k^{\prime}, m^{\prime} I_{1}, n I_{2}\right)\right) \bullet\left(\left(a_{1}, b_{1} I_{1}, c_{1} I_{2}\right)\left(a_{2}, b_{2} I_{1}, c_{2} I_{2}\right)\right)$

$=(\alpha \beta) \bullet u$.

5. $(-\alpha) u=\left\{\left(x, y I_{1}, z I_{2}\right)\left(p, q I_{1}, r I_{2}\right)\right): x \in-k \bullet a_{1}, y \in-k \bullet b_{1} \cup-m \bullet a_{1} \cup-m \bullet b_{1} \cup-m \bullet c_{1} \cup-n \bullet$ $b_{1}, z \in-k \bullet c_{1} \cup-n \bullet a_{1} \cup-n \bullet c_{1}, p \in-k \bullet a_{2}, q \in-k \bullet b_{2} \cup-m \bullet a_{2} \cup-m \bullet b_{2} \cup-m \bullet c_{2} \cup-n \bullet b_{2}, r \in$ $\left.-k \bullet c_{2} \cup-n \bullet a_{2} \cup-n \bullet c_{2}\right\}$

$=\left\{\left(x, y I_{1}, z I_{2}\left(p, q I_{1}, r I_{2}\right)\right): x \in k \bullet\left(-a_{1}\right), y \in k \bullet\left(-b_{1}\right) \cup m \bullet\left(-a_{1}\right) \cup m \bullet\left(-b_{1}\right) \cup m \bullet\left(-c_{1}\right) \cup\right.$ $n \bullet\left(-b_{1}\right), z \in k \bullet\left(-c_{1}\right) \cup n \bullet\left(-a_{1}\right) \cup n \bullet\left(-c_{1}\right), p \in k \bullet\left(-a_{2}\right), q \in k \bullet\left(-b_{2}\right) \cup m \bullet\left(-a_{2}\right) \cup m \bullet$ $\left.\left(-b_{2}\right) \cup m \bullet\left(-c_{2}\right) \cup n \bullet\left(-b_{2}\right), r \in k \bullet\left(-c_{2}\right) \cup n \bullet\left(-a_{2}\right) \cup n \bullet\left(-c_{2}\right)\right\}$

$=\left(k, m I_{1}, n I_{2}\right)\left(-\left(\left(a_{1}, b_{1} I_{1}, c_{1} I_{2}\right)\left(a_{2}, b_{2} I_{1}, c_{2} I_{2}\right)\right)\right)=\alpha(-u)$.

6. $\left.1 \bullet u=\left\{\left(x, y I_{1}, z I_{2}\right)\left(p, q I_{1}, r I_{2}\right)\right): x \in 1 \bullet a_{1}, y \in 1 \bullet b_{1}, z \in 1 \bullet c_{1}, p \in 1 \bullet a_{2}, q \in 1 \bullet b_{2}, r \in 1 \bullet c_{2}\right\}$ $=\left\{\left(a_{1}, b_{1} I_{1}, c_{1} I_{2}\right)\left(a_{2}, b_{2} I_{1}, c_{2} I_{2}\right)\right): a_{1} \in 1 \bullet a_{1}, b_{1} \in 1 \bullet b_{1}, c_{1} \in 1 \bullet c_{1}, a_{2} \in 1 \bullet a_{2}, b_{2} \in 1 \bullet b_{2}, c_{2} \in$ $\left.1 \bullet c_{2}\right\}$, which shows that $u \in 1 \bullet u$.

Accordingly, $V_{1}\left(I_{1}, I_{2}\right) \times V_{2}\left(I_{1}, I_{2}\right)$ is a strong refined neutrosophic hypervector space.

Proposition 2.9. Let $\left(V\left(I_{1}, I_{2}\right), \oplus, \bullet_{1}, K\right)$ and $\left(H,+_{H}, \bullet_{H}, K\right)$ be a weak refined neutrosophic hypervector spaces and a hypervector space, respectively. Let

$V\left(I_{1}, I_{2}\right) \times H=\left\{\left(\left(a, b I_{1}, c I_{2}\right), h\right):\left(a, b I_{1}, c I_{2}\right) \in V_{1}\left(I_{1}, I_{2}\right), h \in H\right\}$.

For all $u=\left(\left(a, b I_{1}, c I_{2}\right), h\right), v=\left(\left(a^{\prime}, b^{\prime} I_{1}, c^{\prime} I_{2}\right), g\right) \in V\left(I_{1}, I_{2}\right) \times H$ and $k \in K$, define:

$u+v=\left(\left(a \oplus a^{\prime},\left(b \oplus b^{\prime}\right) I_{1},\left(c \oplus c^{\prime}\right) I_{2}\right), h+_{H} g\right)$,

$k \bullet u=\left\{\left(\left(x, y I_{1}, z I_{2}\right), p\right): x \in k \bullet_{1} a, y \in k \bullet_{1} b \in k \bullet_{1} c, p \in k \bullet_{H} h\right\}$.

Then $\left(V_{1}\left(I_{1}, I_{2}\right) \times H,+, \bullet, K\right)$ is a weak neutrosophic hypervector space.

Proof. The proof follows from the same pattern as the proof of Proposition 2.8 .

Definition 2.10. Let $\left(V\left(I_{1}, I_{2}\right),+, \bullet, K\left(I_{1}, I_{2}\right)\right)$ be a strong refined neutrosophic hypervector space over a refined neutrosophic field $K\left(I_{1}, I_{2}\right)$ and let $W\left[I_{1}, I_{2}\right]$ be a nonempty subset of $V\left(I_{1}, I_{2}\right) . W\left[I_{1}, I_{2}\right]$ is said to be a subhypervector space of $V\left(I_{1}, I_{2}\right)$ if $\left(W\left[I_{1}, I_{2}\right],+, \bullet, K\left(I_{1}, I_{2}\right)\right)$ is also a refined neutrosophic hypervector space over the refined neutrosophic field $K\left(I_{1}, I_{2}\right)$. It is essential that $W\left[I_{1}, I_{2}\right]$ contains a proper subset which is a hypervector space over a field $K$.

Example 2.11. Let $V\left(I_{1}, I_{2}\right)=\mathbb{R}^{2}\left(I_{1}, I_{2}\right)$ and $K=\mathbb{R}\left(I_{1}, I_{2}\right)$ then $\left(\mathbb{R}^{2}\left(I_{1}, I_{2}\right),+, \bullet, K\left(I_{1}, I_{2}\right)\right)$ is a strong refined neutrosophic hypervector space over refined neutrosophic field $K=R\left(I_{1}, 1_{2}\right)$, where the hyperoperations + and $\bullet$ are defined $\forall u=\left(\left(a_{1}, b_{1} I_{1}, c_{1} I_{1}\right),\left(a_{2}, b_{2} I_{1}, c I_{2}\right)\right)$,

$v=\left(\left(a_{1}^{\prime}, b_{1}^{\prime} I_{1}, c_{1}^{\prime} I_{1}\right),\left(a_{2}^{\prime}, b_{2} I_{1}, c^{\prime} I_{2}\right)\right) \in V\left(I_{1}, I_{2}\right)$ by :

$u+v=\left(\left(a_{1}+a_{1}^{\prime},\left(b_{1}+b_{1}^{\prime}\right) I_{1},\left(c_{1}+c_{1}^{\prime}\right) I_{2}\right),\left(a_{2}+a_{2}^{\prime},\left(b_{2}+b_{2}^{\prime}\right) I_{1},\left(c_{2}+c_{2}^{\prime}\right) I_{2}\right)\right)$, 
$\alpha \bullet u=\left\{\left(\left(x, y I_{1}, z I_{2}\right),\left(p, q I_{1}, r I_{2}\right)\right): x \in k \bullet a_{1}, y \in k \bullet b_{1} \cup m \bullet a_{1} \cup m \bullet b_{1} \cup m \bullet c_{1} \cup n \bullet b_{1}\right.$, $z \in k \bullet c_{1} \cup n \bullet a_{1} \cup n \bullet c_{1}, p \in k \bullet a_{2}, q \in k \bullet b_{2} \cup m \bullet a_{2} \cup m \bullet b_{2} \cup m \bullet c_{2} \cup n \bullet b_{2}$,

$\left.r \in k \bullet c_{2} \cup n \bullet a_{2} \cup n \bullet c_{2}\right\}$.

Let $W\left(I_{1}, I_{2}\right)=K\left(I_{1}, I_{2}\right) \times\left\{\left(0,0 I_{1}, 0 I_{2}\right)\right\} \subseteq V\left(I_{1}, I_{2}\right)$.

Then $W\left(I_{1}, I_{2}\right)$ is a strong refined neutrosophic subhypervector space.

Proof. Since $\theta=\left(\left(0,0 I_{1}, 0 I_{2}\right),\left(0,0 I_{1}, 0 I_{2}\right)\right) \in W\left(I_{1}, I_{2}\right)$. Then $W\left(I_{1}, I_{2}\right) \neq \emptyset$.

Now let $u_{1}=\left(\left(a_{1}, b_{1} I_{1}, c_{2} I_{2}\right),\left(0,0 I_{1}, 0 I_{2}\right)\right), v_{1}=\left(\left(a_{1}^{\prime}, b 1 I_{1}, c_{1} I_{2}\right)\left(0,0 I_{1}, 0 I_{2}\right)\right) \in W\left(I_{1}, I_{2}\right)$, and $\alpha=$ $\left(k, m I_{1}, n I_{2}\right), \beta=\left(k^{\prime}, m^{\prime} I_{1}, n^{\prime} I_{2}\right) \in K\left(I_{1}, I_{2}\right)$ with $a_{1}, b_{1}, c_{1}, a_{1}^{\prime}, b_{1}^{\prime}, c_{1}^{\prime}, k, m, n, k^{\prime}, m^{\prime}, n^{\prime} \in \mathbb{R}$.

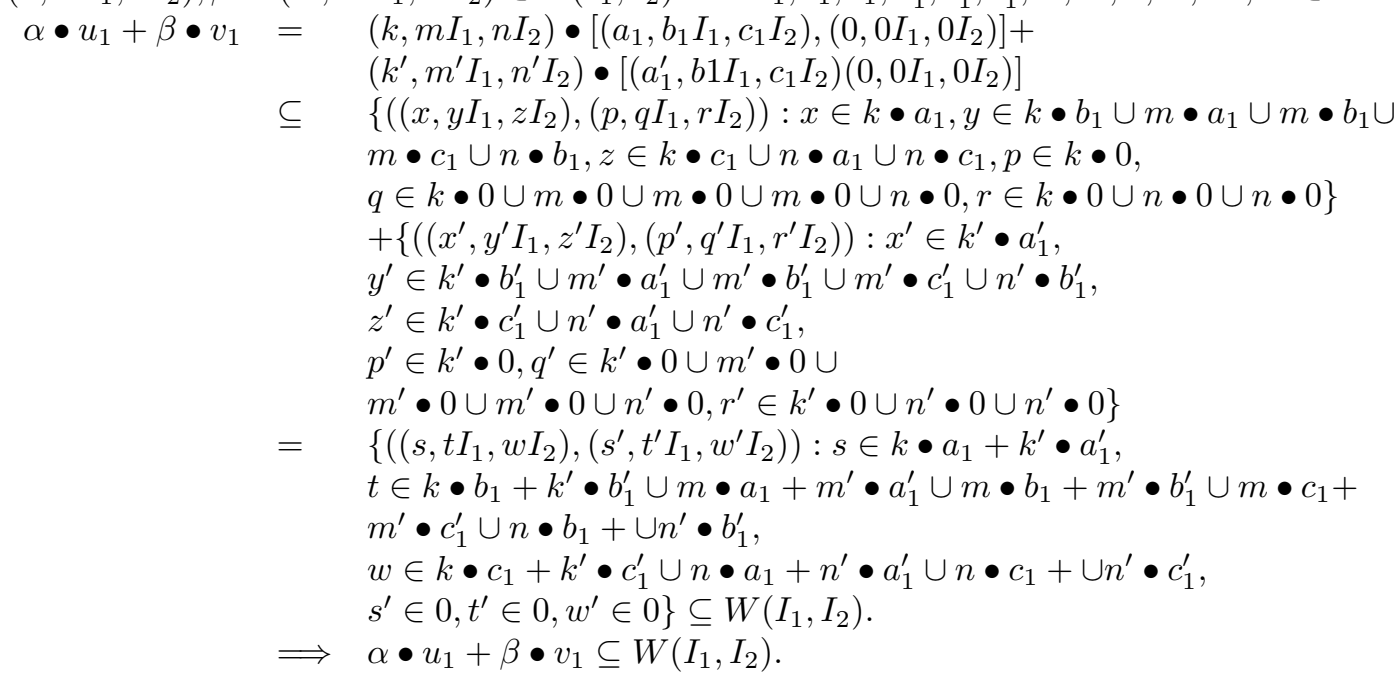

Lastly, we can see from the definition of $W\left(I_{1}, I_{2}\right)$ that $W\left(I_{1}, I_{2}\right)$ contains a proper subset which is a hypervector space over $K$.

To this end we can conclude that $W\left(I_{1}, I_{2}\right)$ is a strong refined neutrosophic hypervector space.

Proposition 2.12. Let $W_{1}\left[I_{1}, I_{2}\right], W_{2}\left[I_{1}, I_{2}\right], \cdots, W_{n}\left[I_{1}, I_{2}\right]$ be refined neutrosophic subhypervector spaces of a strong refined neutrosophic hypervector space $\left(V\left(I_{1}, I_{2}\right),+, \bullet, K\left(I_{1}, I_{2}\right)\right)$ over a refined neutrosophic field $K\left(I_{1}, I_{2}\right)$. Then $\bigcap_{i=1}^{n} W_{i}\left[I_{1}, I_{2}\right]$ is a refined neutrosophic subhypervector space of $V\left(I_{1}, I_{2}\right)$.

Proof. Consider the collection of refined neutrosophic subhypervector space

$\left\{W_{i}\left(I_{1}, I_{2}\right): i=1,2, \cdots n\right\}$ of a strong refined neutrosophic hypervector space $V\left(I_{1}, I_{2}\right)$.

Take $u=\left(a, b I_{1}, c I_{2}\right), v=\left(d, e I_{1}, f I_{2}\right), \alpha=\left(k, p I_{1}, q I_{2}\right)$ and $\beta=\left(r, s I_{1}, t I_{2}\right)$.

Let $u, v \in \bigcap_{i=1}^{n} W_{i}\left(I_{1}, I_{2}\right)$ then $u, v \in W_{i}\left(I_{1}, I_{2}\right)$ for all $i=1,2, \cdots n$.

Now for all scalars $\alpha, \beta \in K\left(I_{1}, I_{2}\right)$ we have that

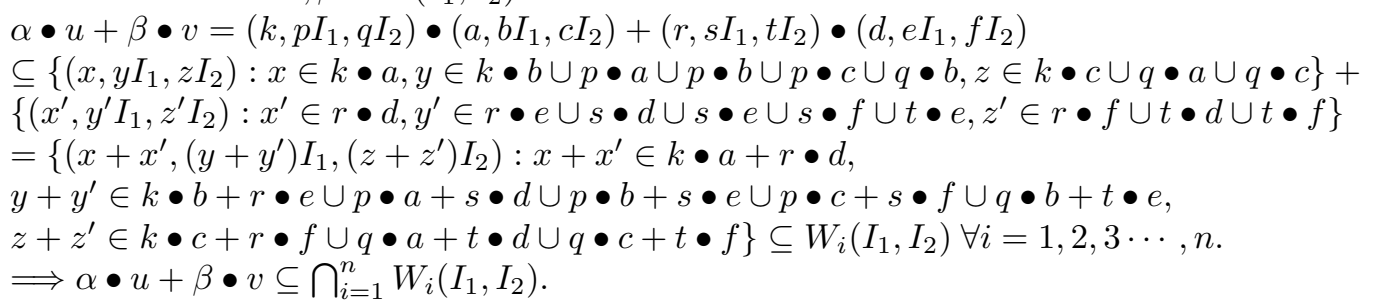

Lastly, since $W_{i}\left(I_{1}, I_{2}\right) \forall i=1,2,3, \cdots, n$ contain proper subsets $W_{i}$ which are hypervector space, $\bigcap_{i=1}^{n} W_{i}\left(I_{1}, I_{2}\right)$ is a strong refined neutrosophic subhyperspace.

Proposition 2.13. Let $W\left[I_{1}, I_{2}\right]$ be a subset of a strong refined neutrosophic hypervector space $\left(V\left(I_{1}, I_{2}\right),+, \bullet, K\left(I_{1}, I_{2}\right)\right)$ over a refined neutrosophic field $K\left(I_{1}, I_{2}\right)$. Then $W\left[I_{1}, I_{2}\right]$ is a refined neutrosophic subhypervector space of $V\left(I_{1}, I_{2}\right)$ if and only if for all $u=\left(a, b I_{1}, c I_{2}\right), v=\left(d, e I_{1}, I_{2}\right) \in V\left(I_{1}, I_{2}\right)$ and $\alpha=\left(k, m I_{1}, n I_{2}\right) \in K\left(I_{1}, I_{2}\right)$, the following conditions hold:
1. $W\left[I_{1}, I_{2}\right] \neq \emptyset$,
2. $u+v \in W\left[I_{1}, I_{2}\right]$,
3. $\alpha \bullet u \subseteq W\left[I_{1}, I_{2}\right]$,

4. $W\left[I_{1}, I_{2}\right]$ contains a proper subset which is a hypervector space over $K$. 
Proposition 2.14. Let $V\left(I_{1}, I_{2}\right)$ be a strong refined neutrosophic hypervector space over $K\left(I_{1}, I_{2}\right)$ and let $U_{1}\left(I_{1}, I_{2}\right), U_{2}\left(I_{1}, I_{2}\right)$ be any strong refined neutrosophic subhypervector spaces of $V\left(I_{1}, I_{2}\right)$.

Then $U_{1}\left(I_{1}, I_{2}\right) \cup U_{2}\left(I_{1}, I_{2}\right)$ is a strong refined neutrosophic subhypervector space if and only if $U_{1}\left(I_{1}, I_{2}\right) \subseteq U_{2}\left(I_{1}, I_{2}\right)$ or $U_{1}\left(I_{1}, I_{2}\right) \supseteq U_{2}\left(I_{1}, I_{2}\right)$.

Proof. Let $U_{1}\left(I_{1}, I_{2}\right)$ and $U_{2}\left(I_{1}, I_{2}\right)$ be any strong refined neutrosophic subhypervector spaces of $V\left(I_{1}, I_{2}\right)$. $\Longrightarrow$ Now, suppose $U_{1}\left(I_{1}, I_{2}\right) \subseteq U_{2}\left(I_{1}, I_{2}\right)$ or $U_{1}\left(I_{1}, I_{2}\right) \supseteq U_{2}\left(I_{1}, I_{2}\right)$ then we shall show the $U_{1}\left(I_{1}, I_{2}\right) \cup U_{2}\left(I_{1}, I_{2}\right)$ is a strong refined neutrosophic subhypervector space of $V\left(I_{1}, I_{2}\right)$.

Without loss of generality, suppose that $U_{1}\left(I_{1}, I_{2}\right) \subseteq U_{2}\left(I_{1}, I_{2}\right)$.

Then we have that $U_{1}\left(I_{1}, I_{2}\right) \cup U_{2}\left(I_{1}, I_{2}\right)=U_{2}\left(I_{1}, I_{2}\right)$. But $U_{2}\left(I_{1}, I_{2}\right)$ is defined to be a strong refined neutrosophic subhypervector space of $V\left(I_{1}, I_{2}\right)$, so $U_{1}\left(I_{1}, I_{2}\right) \cup U_{2}\left(I_{1}, I_{2}\right)$ is a strong refined neutrosophic subhypervector space of $V\left(I_{1}, I_{2}\right)$.

$\Longleftarrow$ We want to show that if $U_{1}\left(I_{1}, I_{2}\right) \cup U_{2}\left(I_{1}, I_{2}\right)$ is a strong refined neutrosophic subhypervector space of $V\left(I_{1}, I_{2}\right)$ then either $U_{1}\left(I_{1}, I_{2}\right) \subseteq U_{2}\left(I_{1}, I_{2}\right)$ or $U_{1}\left(I_{1}, I_{2}\right) \supseteq U_{2}\left(I_{1}, I_{2}\right)$.

Now suppose that $U_{1}\left(I_{1}, I_{2}\right) \cup \bar{U}_{2}\left(I_{1}, I_{2}\right)$ is a strong refined neutrosophic subhypervector space of $V\left(I_{1}, I_{2}\right)$ and suppose by contradiction that $U_{1}\left(I_{1}, I_{2}\right) \nsubseteq U_{2}\left(I_{1}, I_{2}\right)$ or $U_{1}\left(I_{1}, I_{2}\right) \nsupseteq U_{2}\left(I_{1}, I_{2}\right)$.

Thus there exist elements $x_{1}=\left(a_{1}+b_{1} I_{1}+c_{1} I_{2}\right) \in U_{1}\left(I_{1}, I_{2}\right) \backslash U_{2}\left(I_{1}, I_{2}\right)$ and

$x_{2}=\left(a_{2}+b_{2} I_{1}+c_{2} I_{2}\right) \in U_{2}\left(I_{1}, I_{2}\right) \backslash U_{1}\left(I_{1}, I_{2}\right)$. So we have that $x_{1}, x_{2} \in U_{1}\left(I_{1}, I_{2}\right) \cup U_{2}\left(I_{1}, I_{2}\right)$,

since $U_{1}\left(I_{1}, I_{2}\right) \cup U_{2}\left(I_{1}, I_{2}\right)$ is a strong refined neutrosophic subhypervector space, we must have that $x_{1}+x_{2}=x_{3} \in U_{1}\left(I_{1}, I_{2}\right) \cup U_{2}\left(I_{1}, I_{2}\right)$.

Therefore $x_{1}+x_{2}=x_{3} \in U_{1}\left(I_{1}, I_{2}\right)$ or $x_{1}+x_{2}=x_{3} \in U_{2}\left(I_{1}, I_{2}\right)$

$\Longrightarrow x_{2}=x_{3}-x_{1} \in U_{1}\left(I_{1}, I_{2}\right)$ or $x_{1}=x_{3}-x_{2} \in U_{2}\left(I_{1}, I_{2}\right)$ which is a contradiction.

Hence $U_{1}\left(I_{1}, I_{2}\right) \subseteq U_{2}\left(I_{1}, I_{2}\right)$ or $U_{1}\left(I_{1}, I_{2}\right) \supseteq U_{2}\left(I_{1}, I_{2}\right)$ as required.

Remark 2.15. If $W_{1}\left[I_{1}, I_{2}\right]$ and $W_{2}\left[I_{1}, I_{2}\right]$ are refined neutrosophic subhypervector spaces of a strong refined neutrosophic hypervector space $V\left(I_{1}, I_{2}\right)$ over a refined neutrosophic field $K\left(I_{1}, I_{2}\right)$, then generally, $W_{1}\left[I_{1}, I_{2}\right] \cup W_{2}\left[I_{1}, I_{2}\right]$ is not a refined neutrosophic subhypervector space of $V\left(I_{1}, I_{2}\right)$ except if $W_{1}\left[I_{1}, I_{2}\right] \subseteq W_{2}\left[I_{1}, I_{2}\right]$ or $W_{2}\left[I_{1}, I_{2}\right] \subseteq W_{1}\left[I_{1}, I_{2}\right]$.

Definition 2.16. Let $W_{1}\left[I_{1}, I_{2}\right]$ and $W_{2}\left[I_{1}, I_{2}\right]$ be two refined neutrosophic subhypervector spaces of a strong refined neutrosophic hypervector space $\left(V\left(I_{1}, I_{2}\right),+, \bullet, K\left(I_{1}, I_{2}\right)\right)$ over a refined neutrosophic field $K\left(I_{1}, I_{2}\right)$. The sum of $W_{1}\left[I_{1}, I_{2}\right]$ and $W_{2}\left[I_{1}, I_{2}\right]$ denoted by $W_{1}\left[I_{1}, I_{2}\right]+W_{2}\left[I_{1}, I_{2}\right]$ is defined by the set

$$
\bigcup\left\{w+x: w=\left(a, b I_{1}, c I_{2}\right) \in W_{1}\left[I_{1}, I_{2}\right], x=\left(d, e I_{1}, f I_{2}\right) \in W_{2}\left[I_{1}, I_{2}\right]\right\} .
$$

If $W_{1}\left[I_{1}, I_{2}\right] \cap W_{2}\left[I_{1}, I_{2}\right]=\{\theta\}$, then the sum of $W_{1}\left[I_{1}, I_{2}\right]$ and $W_{2}\left[I_{1}, I_{2}\right]$ is denoted by $W_{1}\left[I_{1}, I_{2}\right] \oplus W_{2}\left[I_{1}, I_{2}\right]$ and it is called the direct sum of $W_{1}\left[I_{1}, I_{2}\right]$ and $W_{2}\left[I_{1}, I_{2}\right]$.

Proposition 2.17. Let $W_{1}\left[I_{1}, I_{2}\right]$ and $W_{2}\left[I_{1}, I_{2}\right]$ be two refined neutrosophic subhypervector spaces of a strong refined neutrosophic hypervector space $\left(V\left(I_{1}, I_{2}\right),+, \bullet, K\left(I_{1}, I_{2}\right)\right)$ over a refined neutrosophic field $K\left(I_{1}, I_{2}\right)$.

1. $W_{1}\left[I_{1}, I_{2}\right]+W_{2}\left[I_{1}, I_{2}\right]$ is a refined neutrosophic subhypervector space of $V\left(I_{1}, I_{2}\right)$.

2. $W_{1}\left[I_{1}, I_{2}\right]+W_{2}\left[I_{1}, I_{2}\right]$ is the least refined neutrosophic subhypervector space of $V\left(I_{1}, I_{2}\right)$ containing $W_{1}\left[I_{1}, I_{2}\right]$ and $W_{2}\left[I_{1}, I_{2}\right]$.

Proof. 1. Since $\theta \in W_{1}\left[I_{1}, I_{2}\right]$ and $\theta \in W_{2}\left[I_{1}, I_{2}\right],\{\theta+\theta\} \subseteq W_{1}\left[I_{1}, I_{2}\right]+W_{2}\left[I_{1}, I_{2}\right]$.

So, $\{\theta\} \subseteq W_{1}\left[I_{1}, I_{2}\right]+W_{2}\left[I_{1}, I_{2}\right] \Longrightarrow \theta \in W_{1}\left[I_{1}, I_{2}\right]+W_{2}\left[I_{1}, I_{2}\right]$, therefore $W_{1}\left[I_{1}, I_{2}\right]+W_{2}\left[I_{1}, I_{2}\right]$ is non-empty.

Let $u=\left(a, b I_{1}, c I_{2}\right), v=\left(d, e I_{1}, f I_{2}\right) \in W_{1}\left[I_{1}, I_{2}\right]+W_{2}\left[I_{1}, I_{2}\right]$, then $\exists u_{1}=\left(a_{1}, b_{1} I_{1}, c_{1} I_{2}\right)$, $u_{2}=\left(a_{2}, b_{2} I_{1}, c_{2} I_{2}\right) \in W_{1}\left[I_{1}, I_{2}\right]$ and $v_{1}=\left(d_{1}, e_{1} I_{1}, f_{1} I_{2}\right), v_{2}=\left(d_{2}, e_{2} I_{1}, f_{2} I_{2}\right) \in W_{2}\left[I_{1}, I_{2}\right]$ such that $u \in u_{1}+v_{1}$ and $v \in u_{2}+v_{2}$.

Let $\alpha=\left(k, m I_{1}, n I_{2}\right), \beta=\left(k^{\prime}, m^{\prime} I_{1}, n^{\prime} I_{2}\right) \in K\left(I_{1}, I_{2}\right)$.

Now $\alpha \bullet u+\beta \bullet v \subseteq \alpha \bullet\left(u_{1}+v_{1}\right)+\beta \bullet\left(u_{2}+v_{2}\right)$

$=\left(k, m I_{1}, n I_{2}\right) \bullet\left(a_{1}+d_{1},\left(b_{1}+e_{1}\right) I_{1},\left(c_{1}+e_{1}\right) I_{2}\right)+$

$\left(k^{\prime}, m^{\prime} I_{1}, n^{\prime} I_{2}\right) \bullet\left(a_{2}+d_{2},\left(b_{2}+e_{2}\right) I_{1},\left(c_{2}+e_{2}\right) I_{2}\right)$

$\subseteq\left\{\left(x_{1}, y_{1} I_{1}, z_{1} I_{2}\right): x_{1} \in k \bullet\left(a_{1}+d_{1}\right)\right.$,

$y_{1} \in k \bullet\left(b_{1}+e_{1}\right) \cup m \bullet\left(a_{1}+d_{1}\right) \cup m \bullet\left(b_{1}+e_{1}\right) \cup m \bullet\left(c_{1}+f_{1}\right) \cup n \bullet\left(b_{1}+e_{1}\right)$,

$\left.z_{1} \in k \bullet\left(c_{1}+f_{1}\right) \cup n \bullet\left(a_{1}+d_{1}\right) \cup n \bullet\left(c_{1}+f_{1}\right)\right\}$

$+\left\{\left(x_{2}, y_{2} I_{1}, z_{2} I_{2}\right): x_{2} \in k^{\prime} \bullet\left(a_{2}+d_{2}\right)\right.$,

$y_{2} \in k^{\prime} \bullet\left(b_{2}+e_{2}\right) \cup m^{\prime} \bullet\left(a_{2}+d_{2}\right) \cup m^{\prime} \bullet\left(b_{2}+e_{2}\right) \cup m^{\prime} \bullet\left(c_{2}+f_{2}\right) \cup n^{\prime} \bullet\left(b_{2}+e_{2}\right)$,

$\left.z_{2} \in k^{\prime} \bullet\left(c_{2}+f_{2}\right) \cup n^{\prime} \bullet\left(a_{2}+d_{2}\right) \cup n^{\prime} \bullet\left(c_{2}+f_{2}\right)\right\}$ 


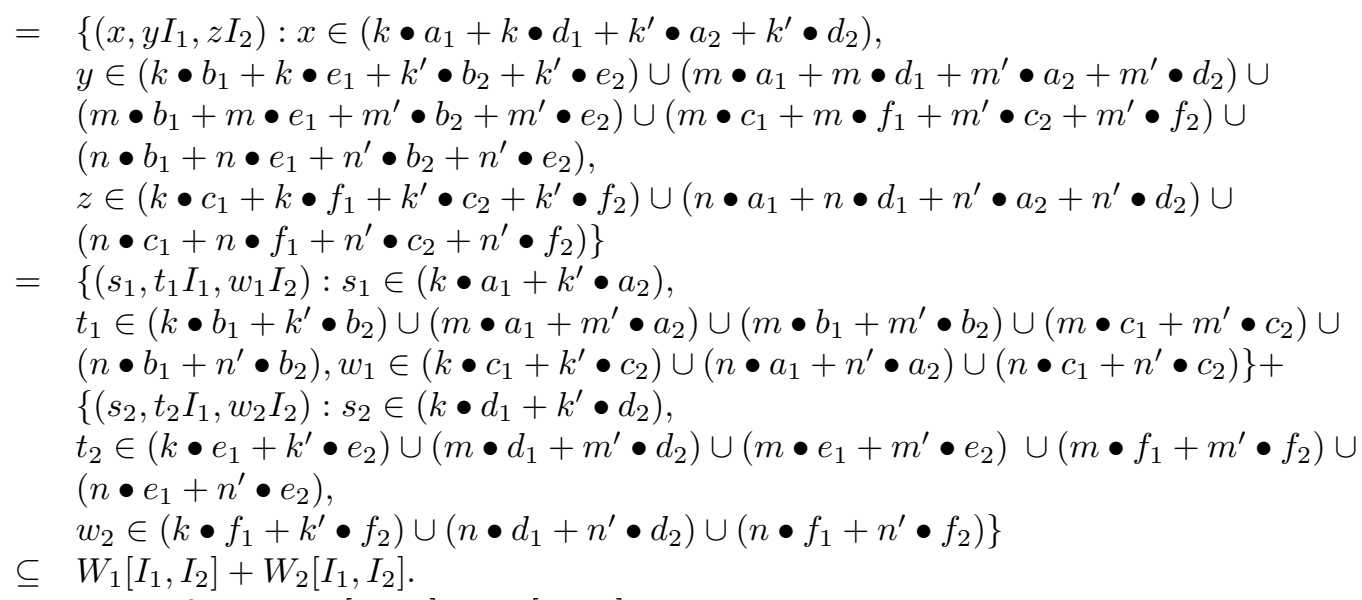

Hence $\alpha \bullet u+\beta \bullet v \subseteq W_{1}\left[I_{1}, I_{2}\right]+W_{2}\left[I_{1}, I_{2}\right]$.

Now since $W_{1}, W_{2}$ are proper subsets of $W_{1}\left[I_{1}, I_{2}\right]$ and $W_{2}\left[I_{1}, I_{2}\right]$ respectively, with both $W_{1}$ and $W_{2}$ being hypervector space. Then $W_{1}+W_{2}$ is a hypervector space which is properly contained in $W_{1}\left[I_{1}, I_{2}\right]+W_{2}\left[I_{1}, I_{2}\right]$. Then we can conclude that $W_{1}\left[I_{1}, I_{2}\right]+W_{2}\left[I_{1}, I_{2}\right]$ is a refined neutrososphic subhypervector space.

2. Let $W\left[I_{1}, I_{2}\right]$ be refined neutrosophic subhypervector space of $V\left[I_{1}, I_{2}\right]$ such that $W_{1}\left[I_{1}, I_{2}\right] \subseteq W\left[I_{1}, I_{2}\right]$ and $W_{2}\left[I_{1}, I_{2}\right] \subseteq W\left[I_{1}, I_{2}\right]$.

Let $u=\left(a, b I_{1}, c I_{2}\right) \in W_{1}\left[I_{1}, I_{2}\right]+W_{2}\left[I_{1}, I_{2}\right]$, then $\exists u_{1}=\left(a_{1}, b_{1} I_{1}, c_{1} I_{2}\right) \in W_{1}\left[I_{1}, I_{2}\right]$ and $u_{2}=\left(a_{2}, b_{2} I_{1}, c_{2} I_{2}\right) \in W_{2}\left[I_{1}, I_{2}\right]$ such that $u \in u_{1}+u_{2}$.

Since $W_{1}\left[I_{1}, I_{2}\right] \subseteq W\left[I_{1}, I_{2}\right]$ and $W_{2}\left[I_{1}, I_{2}\right] \subseteq W\left[I_{1}, I_{2}\right]$, then $u_{1}, u_{2} \in W\left[I_{1}, I_{2}\right]$.

Again since $W\left[I_{1}, I_{2}\right]$ is a refined neutrosophic subhypervector space of $V\left[I_{1}, I_{2}\right]$, then we have that $u_{1}+u_{2} \subseteq W\left[I_{1}, I_{2}\right] \Longrightarrow u \in W\left[I_{1}, I_{2}\right]$.

Hence $W_{1}\left[I_{1}, I_{2}\right]+W_{2}\left[I_{1}, I_{2}\right] \subseteq W\left[I_{1}, I_{2}\right]$ and the proof follows.

Remark 2.18. If $V\left(I_{1}, I_{2}\right)$ is a weak refined neutrosophic strongly left distributive hypervector space over a field $K$, then

1. $W\left[I_{1}, I_{2}\right]=\bigcup\{k \bullet u: k \in K\}$ forms a weak refined neutrosophic subhypervector space of $V\left(I_{1}, I_{2}\right)$, where $u=\left(a, b I_{1}, c I_{2}\right) \in V\left(I_{1}, I_{2}\right)$. This refined neutrosophic subhypervector space is said to be generated by the refined neutrosophic vector $u$ and it is called a refined neutrosophic hyperline span by the refined neutrosophic vector $u$.

2. If $u=\left(a, b I_{1}, c I_{2}\right), v=\left(d, e I_{1}, f I_{2}\right) \in V\left(I_{1}, I_{2}\right)$, then the set $W=\bigcup\{\alpha \bullet u+\beta \bullet v, \alpha, \beta \in K\}$ is a weak refined neutrosophic subhypervector space of $V\left(I_{1}, I_{2}\right)$. This refined neutrosophic subhypervector space is called refined neutrosophic hyperlinear span of the refined neutrosophic vectors $u$ and $v$.

Proposition 2.19. Let $V\left(I_{1}, I_{2}\right)$ be a weak refined neutrosophic strongly left distributive hypervector space over the field $K$ and $u_{1}, u_{2}, \cdots, u_{n} \in V\left(I_{1}, I_{2}\right)$, with $u_{i}=\left(a_{i}, b_{i} I_{1}, c_{i} I_{2}\right)$ for $i=1,2,3 \cdots n$. Then

1. $W\left(I_{1}, I_{2}\right)=\bigcup\left\{\alpha_{1} \bullet u_{1}+\alpha_{2} \bullet u_{2}+\cdots+\alpha_{n} \bullet u_{n}: \alpha_{1}, \alpha_{2}, \cdots, \alpha_{n} \in K\right\}$ is a weak refined neutrosophic subhypervector space of $V\left(I_{1}, I_{2}\right)$.

2. $W\left(I_{1}, I_{2}\right)$ is the smallest weak refined neutrosophic subhypervector space of $V\left(I_{1}, I_{2}\right)$ containing $u_{1}, u_{2}, \cdots, u_{n}$.

Proof. 1. The proof follows from similar approach as 1 of Proposition 2.17.

2. Suppose that $M\left(I_{1}, I_{2}\right)$ is a weak refined neutrosophic subhypervector space of $V\left(I_{1}, I_{2}\right)$ containing $u_{1}=\left(a_{1}, b_{1} I_{1}, c_{1} I_{2}\right), u_{2}=\left(a_{2}, b_{2} I_{1}, c_{2} I_{2}\right), \cdots, u_{n}=\left(a_{n}, b_{n} I_{1}, c_{n} I_{2}\right)$. Let $t \in W\left(I_{1}, I_{2}\right)$, then there exist $\alpha_{1}, \alpha_{2}, \cdots, \alpha_{n} \in K$ such that

$$
t \in \alpha_{1} \bullet\left(a_{1}, b_{1} I_{1}, c_{1} I_{2}\right)+\alpha_{2} \bullet\left(a_{2}, b_{2} I_{1}, c_{2} I_{2}\right)+\cdots+\alpha_{n} \bullet\left(a_{n}, b_{n} I_{1}, c_{n} I_{2}\right) .
$$

Therefore $t \in M\left(I_{1}, I_{2}\right) \Longrightarrow W\left(I_{1}, I_{2}\right) \subseteq M\left(I_{1}, I_{2}\right)$.

Hence $W\left(I_{1}, I_{2}\right)$ is the smallest weak refined neutrosophic subhypervector space of $V\left(I_{1}, I_{2}\right)$ containing $u_{1}, u_{2}, \cdots, u_{n}$. 
Proposition 2.20. Let $V\left(I_{1}, I_{2}\right)$ be a strong refined neutrosophic hypervector space over a refined neutrosophic field $K\left(I_{1}, I_{2}\right)$, and let

$u_{1}=\left(a_{1}, b_{1} I_{1}, c_{1} I_{2}\right), u_{2}=\left(a_{2}, b_{2} I_{1}, c_{2} I_{2}\right), \cdots, u_{n}=\left(a_{n}, b_{n} I_{1}, c_{n} I_{2}\right) \in V\left(I_{1}, I_{2}\right)$,

$\alpha_{1}=\left(k_{1}, m_{1} I_{1}, t_{1} I_{2}\right), \alpha_{2}=\left(k_{2}, m_{2} I_{1}, t_{2} I_{2}\right) \cdots, \alpha_{n}=\left(k_{n}, m_{n} I_{1}, t_{n} I_{2}\right)$.

Then:

1. $W\left(I_{1}, I_{2}\right)=\bigcup\left\{\alpha_{1} \bullet u_{1}+\alpha_{2} \bullet u_{2}+\cdots+\alpha_{n} \bullet u_{n}: \alpha_{1}, \alpha_{2}, \cdots, \alpha_{n} \in K\left(I_{1}, I_{2}\right)\right\}$ is a refined neutrosophic subhypervector space of $V\left(I_{1}, I_{2}\right)$.

2. $W\left(I_{1}, I_{2}\right)$ is the smallest refined neutrosophic subhypervector space of $V\left(I_{1}, I_{2}\right)$ containing $u_{1}, u_{2}, \cdots, u_{n}$.

Proof: The proof follows from similar approach as that of Proposition 2.19 .

Remark 2.21. The refined neutrosophic subhypervector space $W\left(I_{1}, I_{2}\right)$ of the strong refined neutrosophic hypervector space $V\left(I_{1}, I_{2}\right)$ over a refined neutrosophic field $K\left(I_{1}, I_{2}\right)$ of Proposition 2.20 is said to be generated by the refined neutrosophic vectors $u_{1}, u_{2}, \cdots, u_{n}$ and we write $W\left(I_{1}, I_{2}\right)=\operatorname{span}\left\{u_{1}, u_{2}, \cdots, u_{n}\right\}$.

Definition 2.22. Let $\left(V\left(I_{1}, I_{2}\right),+, \bullet, K\left(I_{1}, I_{2}\right)\right)$ be a strong refined neutrosophic hypervector space over a refined neutrosophic field $K\left(I_{1}, I_{2}\right)$ and let

$B\left(I_{1}, I_{2}\right)=\left\{u_{1}=\left(a_{1}, b_{1} I_{1}, c_{1} I_{2}\right), u_{2}=\left(a_{2}, b_{2} I_{1}, c_{2} I_{2}\right), \cdots, u_{n}=\left(a_{n}, b_{n} I_{1}, c_{n} I_{2}\right)\right\}$ be a subset of $V\left(I_{1}, I_{2}\right) . B\left(I_{1}, I_{2}\right)$ is said to generate or span $V\left(I_{1}, I_{2}\right)$ if $V\left(I_{1}, I_{2}\right)=\operatorname{span}\left(B\left(I_{1}, I_{2}\right)\right)$.

Example 2.23. Let $V\left(I_{1}, I_{2}\right)=\mathbb{R}^{3}\left(I_{1}, I_{2}\right)$ be a strong refined neutrosophic hypervector space over a neutrosophic field $R\left(I_{1}, I_{2}\right)$ and let $B\left(I_{1}, I_{2}\right)=\left\{u_{1}=\left(\left(1,0 I_{1}, 0 I_{2}\right),\left(0,0 I_{1}, 0 I_{2}\right),\left(0,0 I_{1}, 0 I_{2}\right)\right)\right.$, $\left.u_{2}=\left(\left(0,0 I_{1}, 0 I_{2}\right),\left(1,0 I_{1}, 0 I_{2}\right),\left(0,0 I_{1}, 0 I_{2}\right)\right), u_{3}=\left(\left(0,0 I_{1}, 0 I_{2}\right),\left(0,0 I_{1}, 0 I_{2}\right),\left(1,0 I_{1}, 0 I_{2}\right)\right)\right\}$.

Then $B\left(I_{1}, I_{2}\right)$ spans $V\left(I_{1}, I_{2}\right)$.

Example 2.24. Let $V\left(I_{1}, I_{2}\right)=\mathbb{R}^{2}\left(I_{1}, I_{2}\right)$ be a weak refined neutrosophic hypervector space over a field $\mathbb{R}$ and let $B\left(I_{1}, I_{2}\right)=\left\{u_{1}=\left(\left(1,0 I_{1}, 0 I_{2}\right),\left(0,0 I_{1}, 0 I_{2}\right)\right), u_{2}=\left(\left(0,0 I_{1}, 0 I_{2}\right),\left(1,0 I_{1}, 0 I_{2}\right)\right)\right.$, $u_{3}=\left(\left(0, I_{1}, 0 I_{2}\right),\left(0,0 I_{1}, 0 I_{2}\right)\right), u_{4}=\left(\left(0,0 I_{1}, 0 I_{2}\right),\left(0, I_{1}, 0 I_{2}\right)\right), u_{5}=\left(\left(0,0 I_{1}, I_{2}\right),\left(0,0 I_{1}, 0 I_{2}\right)\right)$, $\left.u_{6}=\left(\left(0,0 I_{1}, 0 I_{2}\right),\left(0,0 I_{1}, I_{2}\right)\right)\right\}$. Then $B\left(I_{1}, I_{2}\right)$ spans $V\left(I_{1}, I_{2}\right)$.

Definition 2.25. Let $W\left[I_{1}, I_{2}\right]$ and $X\left[I_{1}, I_{2}\right]$ be two refined neutrosophic subhypervector spaces of a strong refined neutrosophic hypervector space $\left(V\left(I_{1}, I_{2}\right),+, \bullet, K\left(I_{1}, I_{2}\right)\right)$ over a refined neutrosophic field $K\left(I_{1}, I_{2}\right)$. $V\left(I_{1}, I_{2}\right)$ is said to be the direct sum of $W\left[I_{1}, I_{2}\right]$ and $X\left[I_{1}, I_{2}\right]$ written $V\left(I_{1}, I_{2}\right)=W\left[I_{1}, I_{2}\right] \oplus X\left[I_{1}, I_{2}\right]$ if every element $v \in V\left(I_{1}, I_{2}\right)$ can be written uniquely as $v=w+x$ where $w \in W\left[I_{1}, I_{2}\right]$ and $x \in X\left[I_{1}, I_{2}\right]$.

Proposition 2.26. Let $W\left[I_{1}, I_{2}\right]$ and $X\left[I_{1}, I_{2}\right]$ be two refined neutrosophic subhypervector spaces of a strong refined neutrosophic hypervector space $\left(V\left(I_{1}, I_{2}\right),+, \bullet, K\left(I_{1}, I_{2}\right)\right)$ over a refined neutrosophic field $K\left(I_{1}, I_{2}\right)$. $V\left(I_{1}, I_{2}\right)=W\left[I_{1}, I_{2}\right] \oplus X\left[I_{1}, I_{2}\right]$ if and only if the following conditions hold:

1. $V\left(I_{1}, I_{2}\right)=W\left[I_{1}, I_{2}\right]+X\left[I_{1}, I_{2}\right]$.

2. $W\left[I_{1}, I_{2}\right] \cap X\left[I_{1}, I_{2}\right]=\{\theta\}$.

Proof. Same as in classical case.

Definition 2.27. Let $\left(V\left(I_{1}, I_{2}\right),+, \bullet, K\left(I_{1}, I_{2}\right)\right)$ be a strong refined neutrosophic hypervector space over a refined neutrosophic field $K\left(I_{1}, I_{2}\right)$. The refined neutrosophic vector

$u=\left(a, b I_{1}, c I_{2}\right) \in V\left(I_{1}, I_{2}\right)$ is said to be a linear combination of the refined neutrosophic vectors $u_{1}=\left(a_{1}, b_{1} I_{1}, c_{1} I_{2}\right), u_{2}=\left(a_{2}, b_{2} I_{1}, c_{2} I_{1}\right), \cdots, u_{n}=\left(a_{n}, b_{n} I_{1}, c_{n} I_{2}\right) \in V\left(I_{1}, I_{2}\right)$ if there exist refined neutrosophic scalars $\alpha_{1}=\left(k_{1}, m_{1} I_{1}, t_{1} I_{2}\right), \alpha_{2}=\left(k_{2}, m_{2} I_{1}, t_{2} I_{2}\right), \cdots, \alpha_{n}=\left(k_{n}, m_{n} I_{1}, t_{n} I_{2}\right) \in K\left(I_{1}, I_{2}\right)$ such that

$$
u \in \alpha_{1} \bullet u_{1}+\alpha_{2} \bullet u_{2}+\cdots+\alpha_{n} \bullet u_{n} .
$$

Definition 2.28. Let $\left(V\left(I_{1}, I_{2}\right),+, \bullet, K\left(I_{1}, I_{2}\right)\right)$ be a strong refined neutrosophic hypervector space over a refined neutrosophic field $K\left(I_{1}, I_{2}\right)$ and let

$B\left(I_{1}, I_{2}\right)=\left\{u_{1}=\left(a_{1}, b_{1} I_{1}, c_{1} I_{2}\right), u_{2}=\left(a_{2}, b_{2} I_{1}, c_{2} I_{2}\right), \cdots, u_{n}=\left(a_{n}, b_{n} I_{1}, c_{n} I_{2}\right)\right\}$ be a subset of $V\left(I_{1}, I_{2}\right)$.

1. $B\left(I_{1}, I_{2}\right)$ is called a linearly dependent set if there exist refined neutrosophic scalars $\alpha_{1}=\left(k_{1}, m_{1} I_{1}, t_{1} I_{2}\right), \alpha_{2}=\left(k_{2}, m_{2} I_{1}, t_{2} I_{2}\right), \cdots, \alpha_{n}=\left(k_{n}, m_{n} I_{1}, t_{n} I_{2}\right)$ (not all zero) such that

$$
\theta \in \alpha_{1} \bullet u_{1}+\alpha_{2} \bullet u_{2}+\cdots+\alpha_{n} \bullet u_{n} .
$$


2. $B\left(I_{1}, I_{2}\right)$ is called a linearly independent set if

$$
\theta \in \alpha_{1} \bullet u_{1}+\alpha_{2} \bullet u_{2}+\cdots+\alpha_{n} \bullet u_{n}
$$

implies that $\alpha_{1}=\alpha_{2}=\cdots=\alpha_{n}=\left(0,0 I_{1}, 0 I_{2}\right)$.

Proposition 2.29. Let $\left(V\left(I_{1}, I_{2}\right),+, \bullet, K\right)$ be a weak refined neutrosophic hypervector space over a field $K$. Any singleton set of non-null refined neutrosophic vector of the weak refined neutrosophic hypervector space $V\left(I_{1}, I_{2}\right)$ is linearly independent.

Proof. Suppose that $\theta \neq v=\left(a, b I_{1}, c I_{2}\right) \in V\left(I_{1}, I_{2}\right)$. Let $\theta \in k \bullet v$ and suppose that $\theta \neq k \in K$.

Then $k^{-1} \in K$ and therefore, $k^{-1} \bullet \theta \subseteq k^{-1} \bullet(k \bullet v)$ so that

$$
\begin{aligned}
\theta & \in\left(k^{-1} k\right) \bullet v \\
& =1 \bullet v \\
& =\left\{\left(x, y I_{1}, z I_{2}\right): x \in 1 \bullet a, y \in 1 \bullet b, z \in 1 \bullet c\right\} \\
& =\left\{\left(x, y I_{1}, z I_{2}\right): x \in\{a\}, y \in\{b\}, z \in\{c\}\right\} \\
& =\left\{\left(a, b I_{1}, c I_{2}\right)\right\} \\
& =\{v\} .
\end{aligned}
$$

This shows that $v=\theta$ which is a contradiction. Hence, $k=\theta$ and thus, the singleton $\{v\}$ is a linearly independent set.

Proposition 2.30. Let $\left(V\left(I_{1}, I_{2}\right),+, \bullet, K\right)$ be a weak refined neutrosophic hypervector space over a field $K$. Any set of refined neutrosophic vectors of the weak refined neutrosophic hypervector space $V\left(I_{1}, I_{2}\right)$ containing the null refined neutrosophic vector is always linearly dependent.

Proposition 2.31. Let $\left(V\left(I_{1}, I_{2}\right),+, \bullet, K\right)$ be a weak refined neutrosophic hypervector space over a field $K$ and let $\mathbb{B}\left(I_{1}, I_{2}\right)=\left\{u_{1}=\left(a_{1}, b_{1} I_{1}, c_{1} I_{2}\right), u_{2}=\left(a_{2}, b_{2} I_{1}, c_{2} I_{2}\right), \cdots, u_{n}=\left(a_{n}, b_{n} I_{1}, c_{n} I_{2}\right)\right\}$ be a subset of $V\left(I_{1}, I_{2}\right)$. Then $B\left(I_{1}, I_{2}\right)$ is a linearly independent set if and only if at least one element of $\mathbb{B}\left(I_{1}, I_{2}\right)$ can be expressed as a linear combination of the remaining elements of $\mathbb{B}\left(I_{1}, I_{2}\right)$.

Proof : This can be easily established.

Proposition 2.32. Let $\left(V\left(I_{1}, I_{2}\right),+, \bullet, K\right)$ be a weak refined neutrosophic hypervector space over a field $K$ and let

$$
B\left(I_{1}, I_{2}\right)=\left\{u_{1}=\left(a_{1}, b_{1} I, c_{1} I_{1}\right), u_{2}=\left(a_{2}, b_{2} I_{1}, c_{2} I_{2}\right), \cdots, u_{n}=\left(a_{n}, b_{n} I_{1}, c I_{2}\right)\right\}
$$

be a subset of $V\left(I_{1}, I_{2}\right)$. Then $B\left(I_{1}, I_{2}\right)$ is a linearly dependent set if and only if at least one element of $B\left(I_{1}, I_{2}\right)$ can be expressed as a linear combination of the remaining elements of $B\left(I_{1}, I_{2}\right)$.

Proof : Suppose that $B\left(I_{1}, I_{2}\right)$ is a linearly dependent set. Then there exist scalars $k_{1}, k_{2}, \cdots, k_{n}$ not all zero in $\mathrm{K}$ such that

$$
\theta \in k_{1} \bullet u_{1}+k_{2} \bullet u_{2}+\cdots+k_{n} \bullet u_{n} .
$$

Suppose that $k_{1} \neq 0$, then $k_{1}^{-1} \in K$ and therefore

$$
\begin{aligned}
k_{1}^{-1} \bullet \theta & \subseteq k_{1}^{-1} \bullet\left(k_{1} \bullet u_{1}+k_{2} \bullet u_{2}+\cdots+k_{n} \bullet u_{n}\right) \\
& =\left(k_{1}^{-1} k_{1}\right) \bullet u_{1}+\left(k_{2}^{-1} k_{2}\right) \bullet u_{2}+\cdots+\left(k_{n}^{-1} k_{n}\right) \bullet u_{n} \\
& =1 \bullet u_{1}+\left(k_{1}^{-1} k_{2}\right) \bullet u_{2}+\cdots+\left(k_{1}^{-1} k_{n}\right) \bullet u_{n}
\end{aligned}
$$

so that

$$
\theta \in 1 \bullet u_{1}+\{u\}
$$

where $u=\left(a, b I_{1}, c I_{2}\right) \in\left(k_{1}^{-1} k_{2}\right) \bullet u_{2}+\cdots+\left(k_{n}^{-1} k_{n}\right) \bullet u_{n}$.

Thus $\theta \in\left\{\left(a+a_{1},\left(b+b_{1}\right) I_{1},\left(c+c_{1}\right) I_{2}\right)\right\}$ from which we obtain $u_{1}=\left(a_{1}, b_{1} I_{1}, c_{1} I_{2}\right)=-u=-\left(a, b I_{1}, c I_{2}\right)$ so that

$$
\begin{aligned}
u_{1} & \in(-1) \bullet u \\
& \subseteq(-1) \bullet\left(\left(k_{1}^{-1} k_{2}\right) \bullet u_{2}+\cdots+\left(k_{n}^{-1} k_{n}\right) \bullet u_{n}\right) \\
& \subseteq\left(-k_{1}^{-1} k_{2}\right) \bullet u_{2}+\left(-k_{1}^{-1} k_{3}\right) \bullet u_{3}+\cdots+\left(-k_{1}^{-1} k_{n}\right) \bullet u_{n} .
\end{aligned}
$$

This shows that $u_{1} \in \operatorname{span}\left\{u_{2}, u_{3}, \cdots, u_{n}\right\}$.

Conversely, suppose that $u_{1} \in \operatorname{span}\left\{u_{2}, u_{3}, \cdots, u_{n}\right\}$ and suppose that $0 \neq-1 \in K$.

Then there exist $k_{2}, k_{3}, \cdots, k_{n} \in K$ such that

$$
u_{1} \in k_{2} \bullet u_{2}+k_{3} \bullet u_{3}+\cdots+k_{n} \bullet u_{n}
$$


and we have

$$
u_{1}+\left(-u_{1}\right) \in(-1) \bullet u_{1}+k_{2} \bullet u_{2}+k_{3} \bullet u_{3}+\bullet+k_{n} \bullet u_{n}
$$

From which

$$
\theta \in(-1) \bullet u_{1}+k_{2} \bullet u_{2}+k_{3} \bullet u_{3}+\cdots+k_{n} \bullet u_{n} .
$$

Since $-1 \neq 0 \in K$, it follows that $B\left(I_{1}, I_{2}\right)$ is a linearly dependent set.

Proposition 2.33. Let $\left(V\left(I_{1}, I_{2}\right),+, \bullet, K\left(I_{1}, I_{2}\right)\right)$ be a strong refined neutrosophic hypervector space over a refined neutrosophic field $K\left(I_{1}, I_{2}\right)$ and let $B_{1}\left(I_{1}, I_{2}\right)$ and $B_{2}\left(I_{1}, I_{2}\right)$ be subsets of $V\left(I_{1}, I_{2}\right)$ such that $B_{1}\left(I_{1}, I_{2}\right) \subseteq B_{2}\left(I_{1}, I_{2}\right)$. If $B_{1}\left(I_{1}, I_{2}\right)$ is linearly dependent, then $B_{2}\left(I_{1}, I_{2}\right)$ is linearly dependent.

Proposition 2.34. Let $\left(V\left(I_{1}, I_{2}\right),+, \bullet, K\left(I_{1}, I_{2}\right)\right)$ be a strong refined neutrosophic hypervector space over a refined neutrosophic field $K\left(I_{1}, I_{2}\right)$ and let $B_{1}\left(I_{1}, I_{2}\right)$ and $B_{2}\left(I_{1}, I_{2}\right)$ be subsets of $V\left(I_{1}, I_{2}\right)$ such that $B_{1}\left(I_{1}, I_{2}\right) \subseteq B_{2}\left(I_{1}, I_{2}\right)$. If $B_{1}\left(I_{1}, I_{2}\right)$ is linearly independent, then $B_{2}\left(I_{1}, I_{2}\right)$ is linearly independent.

Definition 2.35. Let $\left(V\left(I_{1}, I_{2}\right),+, \bullet, K\left(I_{1}, I_{2}\right)\right)$ be a strong refined neutrosophic hypervector space over a refined neutrosophic field $K\left(I_{1}, I_{2}\right)$ and let $B\left(I_{1}, I_{2}\right)=\left\{u_{1}=\left(a_{1}, b_{1} I_{1}, c_{1} I_{1}\right), u_{2}=\left(a_{2}, b_{2} I_{1}, c_{2} I_{2}\right), \cdots\right\}$ be a subset of $V\left(I_{1}, I_{2}\right) . B\left(I_{1}, I_{2}\right)$ is said to be a basis for $V\left(I_{1}, I_{2}\right)$ if the following conditions hold:

1. $B\left(I_{1}, I_{2}\right)$ is a linearly independent set

2. $V\left(I_{1}, I_{2}\right)=\operatorname{span}\left(B\left(I_{1}, I_{2}\right)\right)$.

If $B\left(I_{1}, I_{2}\right)$ is finite and its cardinality is $\mathrm{n}$, then $V\left(I_{1}, I_{2}\right)$ is called an n-dimensional strong refined neutrosophic hypervector space and we write $\operatorname{dim}_{s}\left(V\left(I_{1}, I_{2}\right)\right)=n$. If $B\left(I_{1}, I_{2}\right)$ is not finite, then $V\left(I_{1}, I_{2}\right)$ is called an infinite-dimensional strong refined neutrosophic hypervector space.

Definition 2.36. Let $\left(V\left(I_{1}, I_{2}\right),+, \bullet, K\left(I_{1}, I_{2}\right)\right)$ be a weak refined neutrosophic hypervector space over a field $K$ and let $B\left(I_{1}, I_{2}\right)=\left\{u_{1}=\left(a_{1}, b_{1} I_{1}, c_{1} I_{1}\right), u_{2}=\left(a_{2}, b_{2} I_{1}, c_{2} I_{2}\right), \cdots\right\}$ be a subset of $V\left(I_{1}, I_{2}\right)$. $B\left(I_{1}, I_{2}\right)$ is said to be a basis for $V\left(I_{1}, I_{2}\right)$ if the following conditions hold:

1. $B\left(I_{1}, I_{2}\right)$ is a linearly independent set

2. $V\left(I_{1}, I_{2}\right)=\operatorname{span}\left(B\left(I_{1}, I_{2}\right)\right)$.

If $B\left(I_{1}, I_{2}\right)$ is finite and its cardinality is $\mathrm{n}$, then $V\left(I_{1}, I_{2}\right)$ is called an n-dimensional weak refined neutrosophic hypervector space and we write $\operatorname{dim}_{w}\left(V\left(I_{1}, I_{2}\right)\right)=n$. If $B\left(I_{1}, I_{2}\right)$ is not finite, then $V\left(I_{1}, I_{2}\right)$ is called an infinite-dimensional weak refined neutrosophic hypervector space.

Example 2.37. In Example $2.23 B\left(I_{1}, I_{2}\right)$ is a basis for $V\left(I_{1}, I_{2}\right)$ and $\operatorname{dim}_{s}\left(V\left(I_{1}, I_{2}\right)\right)=3$.

Example 2.38. In Example 2.24 $B\left(I_{1}, I_{2}\right)$ is a basis for $V\left(I_{1}, I_{2}\right)$ and $\operatorname{dim}_{W}\left(V\left(I_{1}, I_{2}\right)\right)=6$.

Proposition 2.39. Let $\left(V\left(I_{1}, I_{2}\right),+, \bullet, K\left(I_{1}, I_{2}\right)\right)$ be a strong refined neutrosophic hypervector space over a refined neutrosophic field $K\left(I_{1}, I_{2}\right)$ and let

$B\left(I_{1}, I_{2}\right)=\left\{u_{1}=\left(a_{1}, b_{1} I_{1}, c_{1} I_{2}\right), u_{2}=\left(a_{2}, b_{2} I_{1}, c_{2} I_{2}\right), \cdots, u_{n}=\left(a_{n}, b_{n} I_{1}, c_{n} I_{2}\right)\right\}$ be a subset of $V\left(I_{1}, I_{2}\right)$. Then $B\left(I_{1}, I_{2}\right)$ is a basis for $V\left(I_{1}, I_{2}\right)$ if and only if each refined neutrosophic vector $u=\left(a, b I_{1}, c I_{2}\right) \in V\left(I_{1}, I_{2}\right)$ can be expressed uniquely as a linear combination of the elements of $B\left(I_{1}, I_{2}\right)$.

Proof. Suppose that each refined neutrosophic vector $u=\left(a, b I, c I_{2}\right) \in V\left(I_{1}, I_{2}\right)$ can be expressed uniquely as a linear combination of the elements of $B\left(I_{1}, I_{2}\right)$. Then $u \in \operatorname{span}\left(B\left(I_{1}, I_{2}\right)\right)=V\left(I_{1}, I_{2}\right)$.

Since such a representation is unique, it follows that $B\left(I_{1}, I_{2}\right)$ is a linearly independent set and since $u \in V\left(I_{1}, I_{2}\right)$ is arbitrary, it follows that $B\left(I_{1}, I_{2}\right)$ is a basis for $V\left(I_{1}, I_{2}\right)$.

Conversely, suppose that $B\left(I_{1}, I_{2}\right)$ is a basis for $V\left(I_{1}, I_{2}\right)$, then $V\left(I_{1}, I_{2}\right)=\operatorname{span}\left(B\left(I_{1}, I_{2}\right)\right)$ and $B\left(I_{1}, I_{2}\right)$ is linearly independent. Now it remains to show that $u=\left(a, b I_{1}, c I_{2}\right) \in V\left(I_{1}, I_{2}\right)$ can be expressed uniquely as a linear combination of the elements of $B\left(I_{1}, I_{2}\right)$.

To this end, for $\alpha_{1}=\left(k_{1}, m_{1} I_{1}, p_{1} I_{2}\right), \alpha_{2}=\left(k_{2}, m_{2} I_{1}, p_{2} I_{2}\right), \cdots, \alpha_{n}=\left(k_{n}, m_{n} I_{1}, p_{n} I_{2}\right)$, $\beta_{1}=\left(r_{1}, s_{1} I_{1}, t_{1} I_{2}\right), \beta_{2}=\left(r_{2}, s_{2} I_{1}, t_{2} I_{2}\right), \cdots, \beta_{n}=\left(r_{n}, s_{n} I_{1}, t_{n} I_{2}\right) \in K\left(I_{1}, I_{2}\right)$, let us express u in two ways as follows:

$$
\begin{aligned}
& u \in \alpha_{1} \bullet u_{1}+\alpha_{2} \bullet u_{2}+\cdots+\alpha_{n} \bullet u_{n}, \\
& u \in \beta_{1} \bullet u_{1}+\beta_{2} \bullet u_{2}+\cdots+\beta_{n} \bullet u_{n} .
\end{aligned}
$$


From equation(2), we have

$$
\begin{aligned}
-u \in(-1) \bullet u & \subseteq(-1) \bullet\left(\beta_{1} \bullet u_{1}+\beta_{2} \bullet u_{2}+\cdots+\beta_{n} \bullet u_{n}\right) \\
& =\left((-1) \beta_{1}\right) \bullet u_{1}+\left((-1) \beta_{2}\right) \bullet u_{2}+\cdots+\left((-1) \beta_{n}\right) \bullet u_{n} \\
& =\left(-\beta_{1}\right) \bullet u_{1}+\left(-\beta_{2}\right) \bullet u_{2}+\bullet+\left(-1 \beta_{n}\right) \bullet u_{n} .
\end{aligned}
$$

From equations (1) and (3), we have

$$
\begin{gathered}
u+(-u) \in\left(\alpha_{1}+\left(-\beta_{1}\right)\right) \bullet u_{1}+\left(\alpha_{2}+\left(-\beta_{2}\right)\right) \bullet u_{2}+\cdots+\left(\alpha_{n}+\left(-\beta_{n}\right)\right) \bullet u_{n} \\
\Longrightarrow \theta \in\left(\alpha_{1}-\beta_{1}\right) \bullet u_{1}+\left(\alpha_{2}-\beta_{2}\right) \bullet u_{2}+\cdots+\left(\alpha_{n}-\beta_{n}\right) \bullet u_{n} .
\end{gathered}
$$

Since $B\left(I_{1}, I_{2}\right)$ is linearly independent, it follows that

$$
\alpha_{1}-\beta_{1}=\alpha_{2}-\beta_{2}=\cdots=\alpha_{n}-\beta_{n}=\left(0,0 I_{1}, 0 I_{2}\right)
$$

and therefore,

$$
\alpha_{1}=\beta_{1}, \alpha_{2}=\beta_{2}, \cdots, \alpha_{n}=\beta_{n} .
$$

This shows that $\mathrm{u}$ has been expressed uniquely as a linear combination of the elements of $B\left(I_{1}, I_{2}\right)$. The proof is complete.

Proposition 2.40. Let $\left(V\left(I_{1}, I_{2}\right),+, \bullet, K\right)$ be a weak refined neutrosophic hypervector space over a field $K$ and let

$B_{1}\left(I_{1}, I_{2}\right)=\left\{u_{1}=\left(a_{1}, b_{1} I_{1}, c_{1} I_{2}\right), u_{2}=\left(a_{2}, b_{2} I_{1}, c_{2} I_{2}\right), \cdots, u_{n}=\left(a_{n}, b_{n} I_{1}, c I_{2}\right)\right\}$ be a linearly independent subset of $V\left(I_{1}, I_{2}\right)$. If $u \in V\left(I_{1}, I_{2}\right) \backslash B_{1}\left(I_{1}, I_{2}\right)=V\left(I_{1}, I_{2}\right) \cap\left(B\left(I_{1}, I_{2}\right)\right)^{c}$ is arbitrary, then $B_{2}\left(I_{1}, I_{2}\right)=\left\{u_{1}=\left(a_{1}, b_{1} I, c_{1} I_{2}\right), u_{2}=\left(a_{2}, b_{2} I_{1}, c_{2} I_{2}\right), \cdots, u_{n}=\left(a_{n}, b_{n} I_{1}, c_{n} I_{2}\right)\right.$, u $\}$ is a linearly dependent set if and only if $u \in \operatorname{span}\left(\left(B_{1}\left(I_{1}, I_{2}\right)\right)\right.$.

Proof. Suppose that $B_{2}\left(I_{1}, I_{2}\right)$ is a linearly dependent set. Then there exist scalars $k_{1}, k_{2}, \cdots, k_{n}, k$ not all zero such that

$$
\theta \in k_{1} \bullet u_{1}+k_{2} \bullet u_{2}+\cdots+k_{n} \bullet u_{n}+k \bullet u .
$$

Suppose that $k=0$, then there exist at least one of the $k_{i}^{\prime} s$ say $k_{1} \neq 0$ and equation (4) becomes

$$
\theta \in k_{1} \bullet u_{1}+k_{2} \bullet u_{2}+\cdots+k_{n} \cdots u_{n}
$$

from which it follows that the set

$B_{1}\left(I_{1}, I_{2}\right)=\left\{u_{1}=\left(a_{1}, b_{1} I_{1}, c_{1} I_{2}\right), u 2=\left(a_{2}, b_{2} I_{1}, c_{2} I_{2}\right), \cdots, u_{n}=\left(a_{n}, b_{n} I_{1}, c I_{2}\right)\right\}$ is linearly dependent. This contradicts the hypothesis that $B_{1}\left(I_{1}, I_{2}\right)$ is linearly independent. Hence $k \neq 0$ and therefore $k^{-1} \in K$. From equation (4), we have

$$
\begin{aligned}
& k^{-1} \bullet \theta \quad \subseteq \\
& \Longrightarrow \theta \quad k^{-1} \bullet\left(k_{1} \bullet u_{1}+k_{2} \bullet u_{2}+\cdots+k_{n} \bullet u_{n}+k \bullet u\right) \\
& \Longrightarrow \theta \quad\left(k^{-1} k_{1}\right) \bullet u_{1}+\left(k^{-1} k_{2}\right) \bullet u_{2}+\cdots+\left(k^{-1} k_{n}\right) \bullet u_{n}+\left(k^{-1} k\right) \bullet u \\
& \Longrightarrow u \quad=-v+u\left(\text { where } v \in\left(k^{-1} k_{1}\right) \bullet u_{1}+\left(k^{-1} k_{2}\right) \bullet u_{2}+\cdots+\left(k^{-1} k_{n}\right) \bullet u_{n}\right) \\
& \Longrightarrow u \quad \in \quad(-1) \bullet\left[\left(k^{-1} k_{1}\right) \bullet u_{1}+\left(k^{-1} k_{2}\right) \bullet u_{2}+\cdots+\left(k^{-1} k_{n}\right) \bullet u_{n}\right] \\
& \Longrightarrow u \quad \in \quad\left(-k^{-1} k_{1}\right) \bullet u_{1}+\left(-k^{-1} k_{2}\right) \bullet u_{2}+\cdots+\left(-k^{-1} k_{n}\right) \bullet u_{n} \\
& \Longrightarrow u \quad \in \quad \operatorname{span}\left(B_{1}\left(I_{1}, I_{2}\right)\right) .
\end{aligned}
$$

Conversely, suppose that $u \in \operatorname{span}\left(B_{1}\left(I_{1}, I_{2}\right)\right)$. Then there exist $k_{1}, k_{2}, \cdots, k_{n} \in K$ such that

$$
\begin{array}{lll}
u & \in & k_{1} \bullet u_{1}+k_{2} \bullet u_{2}+\cdots+k_{n} \bullet u_{n} \\
\Longrightarrow u+(-u) & \in & k_{1} \bullet u_{1}+k_{2} \bullet u_{2}+\cdots+k_{n} \bullet u_{n}+(-1) \bullet u \\
\Longrightarrow \theta & \in & k_{1} \bullet u_{1}+k_{2} \bullet u_{2}+\cdots+k_{n} \bullet u_{n}+(-1) \bullet u .
\end{array}
$$

Since $u \notin B_{1}\left(I_{1}, I_{2}\right)$ and $B_{1}\left(I_{1}, I_{2}\right)$ is linearly independent, it follows that $\left\{u_{1}, u_{2}, \cdots, u_{n}, u\right\}=B_{2}\left(I_{1}, I_{2}\right)$ is a linearly dependent set. The proof is complete.

Definition 2.41. Let $W\left[I_{1}, I_{2}\right]$ be a refined neutrosophic subhypervector space of a strong refined neutrosophic hypervector space $\left(V\left(I_{1}, I_{2}\right),+, \bullet, K\left(I_{1}, I_{2}\right)\right)$ over a refined neutrosophic field $K\left(I_{1}, I_{2}\right)$. The quotient $V\left(I_{1}, I_{2}\right) / W\left[I_{1}, I_{2}\right]$ is defined by the set

$$
\left\{[v]=v+W\left[I_{1}, I_{2}\right]: v \in V\left(I_{1}, I_{2}\right)\right\} .
$$


Proposition 2.42. Let $V\left(I_{1}, I_{2}\right) / W\left[I_{1}, I_{2}\right]=\left\{[v]=v+W\left[I_{1}, I_{2}\right]: v \in V\left(I_{1}, I_{2}\right)\right\}$. If for every $[u],[v] \in V\left(I_{1}, I_{2}\right) / W\left[I_{1}, I_{2}\right]$ and $\alpha \in K\left(I_{1}, I_{2}\right)$ we define:

$$
[u] \oplus[v]=(u+v)+W\left[I_{1}, I_{2}\right]
$$

and

$$
\alpha \odot[u]=[\alpha \bullet u]=\{[x]: x \in \alpha \bullet u\} .
$$

$\left(V\left(I_{1}, I_{2}\right) / W\left[I_{1}, I_{2}\right], \oplus, \odot, K\left(I_{1}, I_{2}\right)\right)$ is a strong refined neutrosophic hypervector space over a refined neutrosophic field $K\left(I_{1}, I_{2}\right)$ called a strong refined neutrosophic quotient hypervector space.

Proof. The proof is similar to the proof in classical case.

Proposition 2.43. Let $W\left[I_{1}, I_{2}\right]$ be a refined neutrosophic subhypervector space of a strong refined neutrosophic hypervector space $V\left(I_{1}, I_{2}\right)$ over a refined neutrosophic field $K\left(I_{1}, I_{2}\right)$, let $\left(V\left(I_{1}, I_{2}\right) / W\left[I_{1}, 1_{2}\right]\right)$ be as defined in Proposition 2.42, then the following hold:

1. $W\left[I_{1}, I_{2}\right]$ is finite dimensional and $\operatorname{dim}_{s} W\left[I_{1}, I_{2}\right] \leq \operatorname{dim}_{s} V\left(I_{1}, I_{2}\right)$.

2. $\operatorname{dim}_{s}\left(V\left(I_{1}, I_{2}\right) / W\left[I_{1}, 1_{2}\right]\right)=\operatorname{dim}_{s} V\left(I_{1}, I_{2}\right)-\operatorname{dim}_{s} W\left[I_{1}, I_{2}\right]$.

Proof:

1. Let $B_{1}\left(I_{1}, I_{2}\right)$ be the basis for $W\left[I_{1}, I_{2}\right]$ and let $B_{2}\left(I_{1}, I_{2}\right)$ be a basis for $V\left(I_{1}, I_{2}\right)$. Since $W\left[I_{1}, I_{2}\right] \subseteq$ $V\left(I_{1}, I_{2}\right)$ then $B_{1}\left(I_{1}, I_{2}\right)$ is contained in $B_{2}\left(I_{1}, I_{2}\right)$. Therefore $B_{1}\left(I_{1}, I_{2}\right)$ is a linearly independent subset of $V\left(I_{1}, I_{2}\right)$. Then we have that

$\left|B_{1}\left(I_{1}, I_{2}\right)\right| \leq\left|B_{2}\left(I_{1}, I_{2}\right)\right|$. Now, since $\left|B_{1}\left(I_{1}, I_{2}\right)\right| \leq\left|B_{2}\left(I_{1}, I_{2}\right)\right|$ and $V\left(I_{1}, I_{2}\right)$ is finite dimensional we can conclude that $W\left(I_{1}, I_{2}\right)$ is finite dimensional and

$$
\operatorname{dim}_{s} W\left(I_{1}, I_{2}\right)=\left|B_{1}\left(I_{1}, I_{2}\right)\right| \leq\left|B_{2}\left(I_{1}, I_{2}\right)\right|=\operatorname{dim}_{s} V\left(I_{1}, I_{2}\right) .
$$

2. Let $\left\{u_{1}, u_{2}, \cdots, u_{m}\right\}$ be a basis of $W\left[I_{1}, I_{2}\right]$. Then this can be filled out to a basis,

$\left\{u_{1}, u_{2} \cdots, u_{m}, v_{1}, v_{2}, \cdots, v_{n}\right\}$ of $V\left(I_{1}, I_{2}\right)$, where $m+n=\operatorname{dim}_{s} V\left(I_{1}, I_{2}\right)$ and $m=\operatorname{dim}_{s} W\left[I_{1}, I_{2}\right]$. Let $\left[v_{1}\right],\left[v_{2}\right], \cdots,\left[v_{n}\right]$ be the images in $V\left(I_{1}, I_{2}\right) / W\left[I_{1}, I_{2}\right]$, of $v_{1}, v_{2}, \cdots, v_{n}$.

Since any vector $v \in V\left(I_{1}, I_{2}\right)$ is in a linear combination of $u_{1}, u_{2}, \cdots, u_{m}, v_{1}, v_{2}, \cdots, v_{n}$, we have that

$$
v \in \alpha_{1} \bullet u_{1}+\alpha_{2} \bullet u_{2}+\cdots+\alpha_{m} \bullet u_{m}+\beta_{1} \bullet v_{1}+\beta_{2} \bullet v_{2}+\cdots+\beta_{n} \bullet v_{n},
$$

then

$v \in\left[\alpha_{1} \bullet u_{1}\right] \oplus\left[\alpha_{2} \bullet u_{2}\right] \oplus \cdots \oplus\left[\alpha_{m} \bullet u_{m}\right] \oplus\left[\beta_{1} \bullet v_{1}\right] \oplus\left[\beta_{2} \bullet v_{2}\right] \oplus \cdots \oplus\left[\beta_{n} \bullet v_{n}\right]$

$\subseteq\left[\beta_{1} \bullet v_{1}\right] \oplus\left[\beta_{2} \bullet v_{2}\right] \oplus \cdots \oplus\left[\beta_{n} \bullet v_{n}\right]\left(\right.$ since $\left.\left[\alpha_{i} \bullet u_{i}\right] \subseteq\left(\alpha_{i} \bullet u_{i}\right)+W\left[I_{1}, I_{2}\right] \subseteq W\left[I_{1}, I_{2}\right]\right)$

$=\beta_{1} \bullet\left[v_{1}\right] \oplus \beta_{2} \bullet\left[v_{2}\right] \oplus \cdots \oplus \beta_{n} \bullet\left[v_{n}\right]$.

Thus $\left[v_{1}\right],\left[v_{2}\right] \cdots,\left[v_{n}\right]$ span $V\left(I_{1}, I_{2}\right) / W\left[I_{1}, I_{2}\right]$. We claim that they are linearly independent, for if

$$
\theta \in \lambda_{1} \bullet\left[v_{1}\right] \oplus \lambda_{2} \bullet\left[v_{2}\right] \oplus \cdots \oplus \lambda_{n} \bullet\left[v_{n}\right]
$$

then

$$
\begin{gathered}
\theta \in \lambda_{1} \bullet\left[v_{1}\right] \oplus \lambda_{2} \bullet\left[v_{2}\right] \oplus \cdots \oplus \lambda_{n} \bullet\left[v_{n}\right] \oplus W\left[I_{1}, I_{2}\right] \\
\theta \subseteq \lambda_{1} \bullet\left[v_{1}\right] \oplus \lambda_{2} \bullet\left[v_{2}\right] \oplus \cdots \oplus \lambda_{n} \bullet\left[v_{n}\right] \oplus \gamma_{1} \bullet\left[u_{1}\right] \oplus \gamma_{2} \bullet\left[u_{2}\right] \oplus \cdots \oplus \gamma_{m} \bullet\left[u_{m}\right]
\end{gathered}
$$

which by the linear independence of the set $\left\{u_{1}, u_{2} \cdots, u_{m}, v_{1}, v_{2} \cdots, v_{n}\right\}$ forces

$\lambda_{1}=\lambda_{2}=\cdots=\lambda_{n}=\gamma_{1}=\gamma_{2}=\cdots=\gamma_{m}=0$.

This shows that $V\left(I_{1}, I_{2}\right) / W\left[I_{1}, I_{2}\right]$ has a basis of $\mathrm{n}$ elements, and

$$
\operatorname{dim}_{s}\left(V\left(I_{1}, I_{2}\right) / W\left[I_{1}, I_{2}\right]\right)=n=(n+m)-m=\operatorname{dim}_{s} V\left(I_{1}, I_{2}\right)-\operatorname{dim}_{s} W\left[I_{1}, I_{2}\right] .
$$

Proposition 2.44. Let $W_{1}\left(I_{1}, I_{2}\right)$ and $W_{2}\left(I_{1}, I_{2}\right)$ be finite dimensional weak refined neutrosophic subhypervector spaces of a weak refined neutrosophic vector space $V\left(I_{1}, I_{2}\right)$ over a field $K$. Then $W_{1}\left(I_{1}, I_{2}\right)+$ $W_{2}\left(I_{1}, I_{2}\right)$ is a finite dimensional refined neutrosophic subhypervector space of $V\left(I_{1}, I_{2}\right)$ and

$$
\begin{aligned}
& \operatorname{dim}_{w}\left(W_{1}\left(I_{1}, I_{2}\right)+W_{2}\left(I_{1}, I_{2}\right)\right)=\operatorname{dim}_{w}\left(W_{1}\left(I_{1}, I_{2}\right)\right)+\operatorname{dim}_{w}\left(W_{2}\left(I_{1}, I_{2}\right)\right)-\operatorname{dim}_{w}\left(W_{1}\left(I_{1}, I_{2}\right) \cap W_{2}\left(I_{1}, I_{2}\right)\right) . \\
& \text { If } V\left(I_{1}, I_{2}\right)=W_{1}\left(I_{1}, I_{2}\right) \oplus W_{2}\left(I_{1}, I_{2}\right) \text { then } \\
& \qquad \operatorname{dim}_{w}\left(W_{1}\left(I_{1}, I_{2}\right)+W_{2}\left(I_{1}, I_{2}\right)\right)=\operatorname{dim}_{w}\left(W_{1}\left(I_{1}, I_{2}\right)\right)+\operatorname{dim}_{w}\left(W_{2}\left(I_{1}, I_{2}\right)\right) .
\end{aligned}
$$


Proof: We know that $W_{1}\left(I_{1}, I_{2}\right) \cap W_{2}\left(I_{1}, I_{2}\right)$ is a refined neutrosophic subhypervector space of both $W_{1}\left(I_{1}, I_{2}\right)$ and $W_{2}\left(I_{1}, I_{2}\right)$. So $W_{1}\left(I_{1}, I_{2}\right) \cap W_{2}\left(I_{1}, I_{2}\right)$ is a finite dimensional refined neutrosophic subhypervector space of $V\left(I_{1}, I_{2}\right)$.

Suppose that $\operatorname{dim}_{w}\left(W_{1}\left(I_{1}, I_{2}\right) \cap W_{2}\left(I_{1}, I_{2}\right)\right)=k, \operatorname{dim}_{w}\left(W_{1}\left(I_{1}, I_{2}\right)\right)=m$ and $\operatorname{dim}_{w}\left(W_{2}\left(I_{1}, I_{2}\right)\right)=n$ then we have that $k \leq m$ and $k \leq n$.

Now, let $\left\{u_{1}, u_{2}, \cdots, u_{k}\right\}$ be a basis of $W_{1}\left(I_{1}, I_{2}\right) \cap W_{2}\left(I_{1}, I_{2}\right)$. Then we have that $\left\{u_{1}, u_{2}, \cdots, u_{k}\right\}$ is a linearly independent set of refined neutrosophic vectors in $W_{1}\left[I_{1}, I_{2}\right]$ and $W_{2}\left[I_{1}, I_{2}\right]$ with $k \leq m$ and $k \leq n$, then it follows that either $\left\{u_{1}, u_{2}, \cdots, u_{k}\right\}$ is a basis of $W_{1}\left[I_{1}, I_{2}\right]$ and $W_{2}\left[I_{1}, I_{2}\right]$ or it can be extended to a basis for $W_{1}\left[I_{1}, I_{2}\right]$ and $W_{2}\left[I_{1}, I_{2}\right]$.

Let $\left\{u_{1}, u_{2}, \cdots, u_{k}, v_{1}, v_{2}, \cdots, v_{m-k}\right\}$ be a basis for $W_{1}\left[I_{1}, I_{2}\right]$, and let $\left\{u_{1}, u_{2}, \cdots, u_{k}, w_{1}, w_{2}, \cdots, w_{n-k}\right\}$ be a basis of $W_{1}\left[I_{1}, I_{2}\right]$.

Then the refined neutrosophic subhypervector space $W_{1}\left[I_{1}, I_{2}\right]+W_{2}\left[I_{1}, I_{2}\right]$ is spanned by the refined neutrosophic vectors $\left\{u_{1}, u_{2}, \cdots, u_{k}, v_{1}, v_{2}, \cdots v_{m-k}, w_{1}, w_{2}, \cdots, w_{n-k}\right\}$ and these refined neutrosophic vectors form an independent set. For suppose

$$
\theta \in \sum_{i=1}^{k} \alpha_{i} u_{i}+\sum_{j=1}^{m} \beta_{j} v_{j}+\sum_{r=1}^{n} \gamma_{r} w_{r} .
$$

Then

$$
\begin{gathered}
-\sum_{r=1}^{n} \gamma_{r} w_{r} \in \sum_{i=1}^{k} \alpha_{i} u_{i}+\sum_{j=1}^{m} \beta_{j} v_{j} \\
\Longrightarrow(-1) \bullet\left(-\sum_{r=1}^{n} \gamma_{r} w_{r}\right) \subseteq \sum_{i=1}^{k}(-1) \bullet \alpha_{i} u_{i}+\sum_{j=1}^{m}(-1) \bullet \beta_{j} v_{j} \\
\Longrightarrow \sum_{r=1}^{n} \gamma_{r} w_{r} \in \sum_{i=1}^{k}\left(-\alpha_{i}\right) u_{i}+\sum_{j=1}^{m}\left(-\beta_{j}\right) v_{j}
\end{gathered}
$$

which shows that $\sum_{r=1}^{n} \gamma_{r} w_{r}$ belongs to $W_{1}\left[I_{1}, I_{2}\right]$. As $\sum_{r=1}^{n} \gamma_{r} w_{r}$ also belongs to $W_{2}\left[I_{1}, I_{2}\right]$, it follows that

$$
\sum_{r=1}^{n} \gamma_{r} w_{r}=\sum_{i=1}^{k} \lambda_{i} u_{i}
$$

for certain scalars $\lambda_{1}, \lambda_{2}, \cdots, \lambda_{k}$.

Because the set $\left\{u_{1}, u_{2}, \cdots, u_{k}, w_{1}, w_{2}, \cdots, w_{n-k}\right\}$ is independent, each of the scalars $\gamma_{r}=0$. Thus

$$
\theta \in \sum_{i=1}^{k} \alpha_{i} u_{i}+\sum_{j=1}^{m} \beta_{j} v_{j}
$$

and since $\left\{u_{1}, u_{2}, \cdots, u_{k}, v_{1}, v_{2}, \cdots, v_{m-k}\right\}$ is also an independent set, each $\alpha_{i}=0$ and each $\beta_{j}=0$. Thus, $\left\{u_{1}, u_{2}, \cdots, u_{k}, v_{1}, v_{2}, \cdots, v_{m-k}, w_{1}, w_{2}, \cdots, w_{n-k}\right\}$ is a basis for $W_{1}\left[I_{1}, I_{2}\right]+W_{2}\left[I_{1}, I_{2}\right]$.

Finally,

$$
\begin{aligned}
& \operatorname{dim}_{w}\left(W_{1}\left(I_{1}, I_{2}\right)+W_{2}\left(I_{1}, I_{2}\right)\right)=k+m-k+n-k \\
& =m+n-k \\
& =\operatorname{dim}_{w}\left(W_{1}\left(I_{1}, I_{2}\right)\right)+\operatorname{dim}_{w}\left(W_{2}\left(I_{1}, I_{2}\right)\right)-\operatorname{dim}_{w}\left(W_{1}\left(I_{1}, I_{2}\right) \cap W_{2}\left(I_{1}, I_{2}\right)\right) .
\end{aligned}
$$

Definition 2.45. Let $\left(V\left(I_{1}, I_{2}\right),+, \bullet, K\left(I_{1}, I_{2}\right)\right)$ and $\left.W\left(I_{1}, I_{2}\right),+^{\prime}, \bullet^{\prime}, K\left(I_{1}, I_{2}\right)\right)$ be two strong refined neutrosophic hypervector spaces over a neutrosophic field $K\left(I_{1}, I_{2}\right)$.

A mapping $\phi: V\left(I_{1}, I_{2}\right) \longrightarrow W\left(I_{1}, I_{2}\right)$ is called a strong refined neutrosophic hypervector space homomorphism if the following conditions hold:

1. $\phi$ is a strong hypervector space homomorphism.

2. $\phi\left(0, I_{1}, I_{2}\right)=\left(0, I_{1}, I_{2}\right)$.

If in addition $\phi$ is a bijection, we say that $V\left(I_{1}, I_{2}\right)$ is isomorphic to $W\left(I_{1}, I_{2}\right)$ and we write $V\left(I_{1}, I_{2}\right) \cong W\left(I_{1}, I_{2}\right)$. 
Proposition 2.46. Let $\left(V\left(I_{1}, I_{2}\right),+, \bullet, K\left(I_{1}, I_{2}\right)\right)$ and $\left(W\left(I_{1}, I_{2}\right),+, \bullet, K\left(I_{1}, I_{2}\right)\right)$ be two strong refined neutrosophic hypervector spaces over a refined neutrosophic field $K\left(I_{1}, I_{2}\right)$ and let $\phi: V\left(I_{1}, I_{2}\right) \longrightarrow W\left(I_{1}, I_{2}\right)$ be a bijective strong refined neutrosophic hypervector space homomorphism.

If $B\left(I_{1}, I_{2}\right)=\left\{u_{1}=\left(a_{1}, b_{1} I_{1}, c_{1} I_{2}\right), u_{2}=\left(a_{2}, b_{2} I_{1}, c_{2} I_{2}\right), \cdots, u_{n}=\left(a_{n}, b_{n} I_{1}, c_{n} I_{n}\right)\right\}$ is a basis for $V\left(I_{1}, I_{2}\right)$, then $B^{\prime}\left(I_{1}, I_{2}\right)=\phi\left(B\left(I_{1}, I_{2}\right)\right)=\left\{\phi\left(u_{1}\right), \phi\left(u_{2}\right), \cdots, \phi\left(u_{n}\right)\right\}$ is a basis for $W\left(I_{1}, I_{2}\right)$.

Proof. Suppose that $B\left(I_{1}, I_{2}\right)$ is a basis for $V\left(I_{1}, I_{2}\right)$. Then for an arbitrary $u=\left(a, b I_{1}, c I_{2}\right) \in V\left(I_{1}, I_{2}\right)$, there exist refined neutrosophic scalars

$\alpha_{1}=\left(k_{1}, m_{1} I_{1}, t_{1} I_{2}\right), \alpha_{2}=\left(k_{2}, m_{2} I_{1}, t_{2} I_{2}\right), \cdots, \alpha_{n}=\left(k_{n}, m_{n} I_{1}, t_{n} I_{2}\right) \in K\left(I_{1}, I_{2}\right)$ such that

$$
\begin{gathered}
u \in \alpha_{1} \bullet u_{1}+\alpha_{2} \bullet u_{2}+\cdots+\alpha_{n} \bullet u_{n} \\
\Longrightarrow \phi(u) \in \phi\left(\alpha_{1} \bullet u_{1}+\alpha_{2} \bullet u_{2}+\cdots+\alpha_{n} \bullet u_{n}\right) \\
=\alpha_{1} \bullet^{\prime} \phi\left(u_{1}\right)+{ }^{\prime} \alpha_{2} \bullet^{\prime} \phi\left(u_{2}\right)+{ }^{\prime} \cdots+{ }^{\prime} \alpha_{n} \bullet^{\prime} \phi\left(u_{n}\right) .
\end{gathered}
$$

Since $\phi$ is surjective, it follows that $\phi(u), \phi\left(u_{1}\right), \phi\left(u_{2}\right), \cdots, \phi\left(u_{n}\right) \in W\left(I_{1}, I_{2}\right)$ and therefore $\phi(u) \in \operatorname{span}\left(B^{\prime}\left(I_{1}, I_{2}\right)\right)$. To complete the proof, we must show that $B^{\prime}\left(I_{1}, I_{2}\right)$ is linearly independent. To this end, suppose that

$$
\phi(\theta) \in \beta_{1} \bullet^{\prime} \phi\left(u_{1}\right)+{ }^{\prime} \beta_{2} \bullet^{\prime} \phi\left(u_{2}\right)+{ }^{\prime} \cdots+^{\prime} \beta_{n} \bullet^{\prime} \phi\left(u_{n}\right)
$$

where $\beta_{1}=\left(p_{1}, q_{1} I_{1}, s_{1} I_{2}\right), \beta_{2}=\left(p_{2}, q_{2} I_{1}, s_{2} I_{2}\right), \cdots, \beta_{n}=\left(p_{n}, q_{n} I_{1}, s_{n} I_{2}\right) \in K\left(I_{1}, I_{2}\right)$, then

$$
\begin{aligned}
\phi(\theta) & \in \phi\left(\beta_{1} \bullet u_{1}\right)+^{\prime} \phi\left(\beta_{2} \bullet u_{2}\right)+^{\prime} \bullet+^{\prime} \phi\left(\beta_{n} \bullet u_{n}\right) \\
& =\phi\left(\beta_{1} \bullet u_{1}+\beta_{2} \bullet u_{2}+\cdots+\beta_{n} \bullet u_{n}\right) .
\end{aligned}
$$

Since $\phi$ is injective, we must have

$$
\theta \in \beta_{1} \bullet u_{1}+\beta_{2} \bullet u_{2}+\cdots+\beta_{n} \bullet u_{n} .
$$

Also, since $B\left(I_{1}, I_{2}\right)$ is linearly independent, we must have $\beta_{1}=\beta_{2}=\cdots=\beta_{n}=\left(0,0 I_{1}, 0 I_{2}\right)$.

Hence $B^{\prime}\left(I_{1}, I_{2}\right)=\left\{\phi\left(u_{1}\right), \phi\left(u_{2}\right), \cdots, \phi\left(u_{n}\right)\right\}$ is linearly independent and therefore a basis for $W\left(I_{1}, I_{2}\right)$.

Remark 2.47. Suppose we wish to transform a refined neutrosophic hypervector space into a neutrosophic hypervector space, an interesting question to ask will be, can we find a mapping that will help us achieve this? The answer to this is Yes.

The mapping $\phi: V\left(I_{1}, I_{2}\right) \longrightarrow V(I)$ defined by

$$
\phi\left(\left(x, y I_{1}, z I_{2}\right)\right)=(x,(y+z) I) \forall x, y, z \in V
$$

will make such transformation possible. This mapping is a non-neutrosophic one. This make sense since every refined neutrosophic hypervector space and neutrosophic hypervector spaces are hypervector spaces.

Proposition 2.48. Let $\left(V\left(I_{1}, I_{2}\right),+, \bullet\right)$ be a weak refined neutrosophic vector space over a field $K$ and let $V(I)$ be a weak neutrosophic vector space over $K$. The mapping $\phi: V\left(I_{1}, I_{2}\right) \longrightarrow V(I)$ defined by

$$
\phi\left(\left(x, y I_{1}, z I_{2}\right)\right)=(x,(y+z) I) \forall x, y, z \in V
$$

is a good linear transformation.

Proof. $\phi$ is well defined. Suppose $\left(x, y I_{1}, z I_{2}\right)=\left(x^{\prime} y^{\prime} I_{1}, z^{\prime} I_{2}\right)$ then we that $x=x^{\prime}, y=y^{\prime}$ and $z^{\prime}=z^{\prime}$. So,

$$
\phi\left(\left(x, y I_{1}, z I_{2}\right)\right)=(x,(y+z) I)=x^{\prime}+\left(y^{\prime}+z^{\prime}\right) I=\phi\left(x^{\prime}, y^{\prime} I_{1}, z^{\prime} I_{2}\right) .
$$

Now, suppose $\left(x, y I_{1}, z I_{2}\right),\left(x^{\prime}, y^{\prime} I_{1}, 1 z^{\prime} I_{2}\right) \in V\left(I_{1}, I_{2}\right)$ then

$$
\begin{aligned}
\phi\left(\left(x, y I_{1}, z I_{2}\right)+\left(x^{\prime}, y^{\prime} I_{1}, z^{\prime} I_{2}\right)\right) & =\phi\left(\left(x+x^{\prime}\right),\left(y+y^{\prime}\right) I_{1},\left(z+z^{\prime}\right) I_{2}\right) \\
& =\left(x+x^{\prime}\right),\left(y+y^{\prime}+z+z^{\prime}\right) I \\
& =\left(x+x^{\prime}\right),\left((y+z)+\left(y^{\prime}+z^{\prime}\right)\right) I \\
& =\left(x+x^{\prime}\right),\left((y+z) I+\left(y^{\prime}+z^{\prime}\right) I\right) \\
& =(x,(y+z) I)+\left(x^{\prime},\left(y^{\prime}+z^{\prime}\right) I\right) \\
& =\phi\left(x, y I_{1}, z I_{2}\right)+\phi\left(x, y I_{1}, z I_{2}\right) .
\end{aligned}
$$




$$
\begin{aligned}
\phi\left(k \circ\left(x, y I_{1}, z I_{2}\right)\right) & =\phi\left\{\left(a, b I_{1}, c I_{2}\right): a \in k \circ x, b \in k \circ y, c \in k \circ z\right\} \\
& =\left\{\phi\left(a, b I_{1}, c I_{2}\right): a \in k \circ x, b \in k \circ y, c \in k \circ z\right\} \\
& =\{(u, v I): u \in a, v \in b+c\} \\
& =\{(u, v I): u \in k \circ x, v \in k \circ y+k \circ z\} \\
& =\{(u, v I): u \in k \circ x, v \in k \circ(y+z)\} \\
& =k \circ(x,(y+z) I) \\
& =k \circ \phi\left(x, y I_{1}, z I_{2}\right) .
\end{aligned}
$$

Hence $\phi$ is a good linear transformation.

Proposition 2.49. Let $L_{k}\left(V\left(I_{1}, I_{2}\right), V(I)\right)$ be the set of good linear transformation from a weak refined neutrosophic vector space $V\left(I_{1}, I_{2}\right)$ over a field $K$ into a weak neutrosophic vector space $V(I)$ over a field $K$. Define addition and scalar multiplication as below;

$$
(\phi+\psi)\left(x, y I_{1}, z I_{2}\right)=\phi\left(\left(x, y I_{1}, z I_{2}\right)\right)+\psi\left(\left(x, y I_{1}, z I_{2}\right)\right)
$$

and for $k \in K$

$$
(k \phi)\left(\left(x, y I_{1}, z I_{2}\right)\right)=k \phi\left(x, y I_{1}, z I_{2}\right) .
$$

Then, it can be shown that $\left(L_{k}\left(V\left(I_{1}, I_{2}\right), V(I)\right),+, \cdot\right)$ is a weak neutrosophic strongly distributive hypervector space.

Definition 2.50. Let $\phi: V\left(I_{1}, I_{2}\right) \longrightarrow V(I)$ be a good linear transformation, then

$$
\begin{aligned}
\operatorname{ker} \phi & =\left\{\left(x, y I_{1}, z I_{2}\right): \phi\left(\left(x, y I_{1}, z I_{2}\right)\right)=(0,0 I)\right\} \\
& =\left\{\left(x, y I_{1}, z I_{2}\right):(x,(y+z) I)=(0,0 I)\right\} \\
& =\left\{\left(0, y I_{1},(-y) I_{2}\right)\right\}
\end{aligned}
$$

Proposition 2.51. Let $\phi: V\left(I_{1}, I_{2}\right) \longrightarrow V(I)$ be a good linear transformation.

1. $\operatorname{ker} \phi$ is a subhyperspace of $V\left(I_{1}, I_{2}\right)$.

2. If $W\left[I_{1}, I_{2}\right]$ is a refined neutrosophic subhyperspace of $V\left(I_{1}, I_{2}\right)$, then the image of $W\left[I_{1}, I_{2}\right], \phi\left(W\left[I_{1}, I_{2}\right]\right)$ is a neutrosophic subhyperspace of $V(I)$.

\section{Conclusion}

This paper studied refinement of neutrosophic hypervector space, linear dependence, independence, bases and dimension of refined neutrosophic hypervector spaces and presented some of their basic properties. Also, the paper established the existence of a good linear transformation between a weak refined neutrosophic hypervector space $V\left(I_{1}, I_{2}\right)$ and a weak neutrosophic hypervector space $V(I)$. We hope to present and study more properties of refined neutrosophic Hypervector spaces in our future papers.

\section{Acknowledgment}

The Authors wish to thank the anonymous reviewers for their valuable comments and suggestions which have been used for the improvement of the paper.

\section{References}

[1] Adeleke, E.O, Agboola, A.A.A and Smarandache, F. Refined Neutrosophic Rings I, International Journal of Neutrosophic Science (IJNS), Vol. 2(2), pp. 77-81, 2020.

[2] Adeleke, E.O, Agboola, A.A.A and Smarandache, F. Refined Neutrosophic Rings II, International Journal of Neutrosophic Science (IJNS), Vol. 2(2), pp. 89-94, 2020.

[3] Agboola, A.A.A., Ibrahim, A.M. and Adeleke, E.O, Elementary Examination of NeutroAlgebras and AntiAlgebras Viz-a-Viz the Classical Number Systems, Vol. 4, pp. 16-19, 2020.

[4] Agboola, A.A.A. On Refined Neutrosophic Algebraic Structures, Neutrosophic Sets and Systems 10, pp 99-101, 2015. 
[5] Agboola, A.A.A. and Akinleye, S.A., Neutrosophic Hypervector Spaces, ROMAI Journal, Vol.11, pp. 1-16, 2015.

[6] Agboola,A.A.A. and Akinleye, S.A., Neutrosophic Vector Spaces, Neutrosophic Sets and Systems 4, pp 9-18, 2014.

[7] Agboola, A.A.A. and Davvaz, B., Introduction to Neutrosophic Hypergroups, Romai J.,Vol. 9(2), pp. 1-10, 2013.

[8] Agboola, A.A.A and Davvaz, B., On Neutrosophic Canonical Hypergroups and Neutrosophic Hyperrings, Neutrosophic Sets and Systems. Vol. 2, pp.34-41, 2014.

[9] Asokkumar, A., Hyperlattice formed by the idempotents of a hyperring, Tamkang J. Math., Vol. 3(38), pp. 209-215, 2007.

[10] Asokkumar, A. and Veelrajan, M., Characterizations of regular hyperrings, Italian J. Pure and App. Math., Vol. 22, pp.115-124, 2007.

[11] Asokkumar, A. and Veelrajan, M., Hyperrings of matrices over a regular hyperring, Italian J. Pure and App. Math. Vol. 23, pp. 113-120, 2008.

[12] Ameri. R. and Dehghan, O.R., On Dimension of Hypervector Spaces, European Journal of Pure and Applied Mathematics, Vol. 1(2), pp. 32-50, 2008.

[13] Atanassov, K., Intuitionistic fuzzy sets, Fuzzy Sets and Systems, Vol. 20, pp. 87-96, 1986.

[14] Bera, T. and Mahapatra,N.K., Introduction to neutrosophic soft groups, Neutrosophic Sets and Systems, Vol. 13, pp. 118-127, 2016, doi.org/10.5281/zenodo.570845.

[15] Bera, T. and Mahapatra, N.K., On neutrosophic normal soft groups, International Journal of Applied and Computational Mathematics., Vol. 3, pp. 3047-3066, 2017. DOI 10.1007/s40819-016-0284-2.

[16] Bera, T. and Mahapatra, N.K., On neutrosophic soft rings, OPSEARCH, Vol. 54, pp. 143-167, 2017. DOI 10.1007/ s12597-016-0273-6.

[17] Bera, T and Mahapatra, N. K., On neutrosophic soft linear spaces, Fuzzy Information and Engineering, Vol. 9, pp 299-324, 2017.

[18] Bera, T and Mahapatra, N. K., On neutrosophic soft field, IJMTT, Vol. 56(7), pp. 472-494, 2018.

[19] Bonansinga, P., Quasicanonical hypergroups, (Italian), Atti Soc. Peloritana Sci. Fis. Mat. Natur., Vol. 27, pp. 9-17, 1981.

[20] Bonansinga, P., Weakly quasicanonical hypergroups, (Italian), Atti Sem. Mat. Fis. Univ. Modena, Vol.30, pp. 286-298, 1981.

[21] Corsini, P., Prolegomena of Hypergroup Theory, Second edition, Aviani editor, (1993).

[22] Corsini, P., Finite canonical hypergroups with partial scalar identities, (Italian), Rend. Circ. Mat. Palermo (2), Vol.36, pp.205-219, 1987.

[23] Davvaz, B. and Leoreanu-Fotea, V., Hyperring Theory and Applications, International Academic Press,Palm Harber, USA, 2007.

[24] De Salvo, M., Hyperrings and hyperfields, Annales Scientifiques de l'Universite de Clermont-Ferrand II, Vol.22, pp. 89-107, 1984.

[25] Marty, F. Role de la notion dhypergroupe dans letude des groupes non abeliens, Comptes Renclus Acad. Sci. Paris Math, Vol. 201, pp 636-638, 1935.

[26] Krasner, M., A class of hyperrings and hyperfields, International Journal of Mathematics and Mathematical Science, Vol 6(2), pp. 307-312, 1983.

[27] Mittas, J., Hypergroupes canoniques, Math. Balkanica, Vol.2, pp. 165-179, 1972.

[28] Muthusamy Velrajan and Arjunan Asokkumar, Note on Isomophism Theorems of Hyperrings, Int. J. Math. Math. Sc., Hindawi Publishing Corporation, pp.1-12, 2010. 
[29] Nakassis, A., Recent results in hyperring and hyperfield theory, Internat. J. Math.Math. Sci., Vol.11(2), pp. 209-220, 1988.

[30] Sanjay Roy and Samanta,T.K., A Note on Hypervector Spaces, Discussiones Mathematicae -General Algebra and Applications Vol. 31(1), pp. 75-99, 2011. < http ://eudml.org/doc/276586 > .

[31] Scafati Tallini,M., Characterization of Remarkable Hypervector spaces, Proc. of 8th int. Congress on Algebraic Hyperstructures and Applications, Samotraki, Greece, Sept. 1-9-2002, Spanidis Press, Xanthi, Greece, ISBN 960-87499-5-6, pp. 231-237, 2003.

[32] Serafimidis, K., Sur les hypergroups canoniques ordonne's et strictement ordonne's,(French) [Ordered and strictly ordered canonical hypergroups], Rend. Mat., Vol. 7(6), pp. 231-238. 1986.

[33] Serafimidis, K., Konstantinidou, M. and Mittas, J., Sur les hypergroups canoniques strictement re'ticule's, (French) [On strictly lattice-ordered canonical hypergroups], Riv. Mat. Pura Appl., Vol. 2, pp 21-35, 1987.

[34] Spartalis, S., A class of hyperrings, Riv. Mat. Pura Appl., Vol. 4, pp.55-64, 1989.

[35] Smarandache, F., A Unifying Field in Logics: Neutrosophic Logic, Neutrosophy, Neutrosophic Set, Neutrosophic Probability, American Research Press, Rehoboth, 2003.

[36] Smarandache, F., (T,I,F)- Neutrosophic Structures, Neutrosophic Sets and Systems, Vol. 8, pp. 3-10, 2015.

[37] Vasantha Kandasamy W.B and Smarandache,F., Basic Neutrosophic Algebraic Structures and Their Applications to Fuzzy and Neutrosophic Models, Hexis, Church Rock,(2004), http://fs.gallup.unm.edu/eBook-otherformats.htm

[38] Vasantha Kandasamy, W.B. and Florentin Smarandache, Some Neutrosophic Algebraic Structures and Neutrosophic N-Algebraic Structures, Hexis, Phoenix, Arizona, (2006), http://www.gallup.unm.edu/ smarandache/eBooks-otherformats.htm.

[39] Vasantha Kandasamy, W.B., Neutrosophic Rings, Hexis, Phoenix, Arizona,(2006) http://www.gallup.unm.edu/ smarandache/eBooks-otherformats.htm.

[40] Wadei Al-Omeri and Smarandache, F., New Neutrosophic Set via Neutrosophic Topological Spaces. Excerpt from Neutrosophic Operation Research Vol I, Pons Editions: Brussels, Belgium, pp. 189-209, 2017.

[41] Wadei Al-Omeri, Neutrosophic crisp Sets Via Neutrosophic crisp Topological Spaces, Neutrosophic Set and Systems Vol 13, pp 96- 104, 2016.

[42] Wadei Al-Omeri and Saeid Jafari, On Generalized Closed Sets and Generalized Pre-Closed Sets in Neutrosophic Topological Spaces, Mathematics, Vol 7, pp 1- 12, 2019. Doi: doi.org/10.3390/math/7010001.

[43] Zadeh, L.A., Fuzzy Sets, Information and Control, Vol. 8, pp. 338-353, 1965. 